\title{
Microglial activation underlies cerebellar deficits produced by repeated cannabis exposure
}

Laura Cutando, ${ }^{1}$ Arnau Busquets-Garcia, ${ }^{1}$ Emma Puighermanal, ${ }^{1}$ Maria Gomis-González, ${ }^{1}$ José María Delgado-García, ${ }^{2}$ Agnès Gruart, ${ }^{2}$ Rafael Maldonado, ${ }^{1}$ and Andrés Ozaita1

1'Laboratori de Neurofarmacologia, Facultat de Ciències de la Salut i de la Vida, Universitat Pompeu Fabra, Barcelona, Spain. 2División de Neurociencias, Universidad Pablo de Olavide, Sevilla, Spain.

\begin{abstract}
Chronic cannabis exposure can lead to cerebellar dysfunction in humans, but the neurobiological mechanisms involved remain incompletely understood. Here, we found that in mice, subchronic administration of the psychoactive component of cannabis, delta9-tetrahydrocannabinol (THC), activated cerebellar microglia and increased the expression of neuroinflammatory markers, including IL-1 $\beta$. This neuroinflammatory phenotype correlated with deficits in cerebellar conditioned learning and fine motor coordination. The neuroinflammatory phenotype was readily detectable in the cerebellum of mice with global loss of the CB1 cannabinoid receptor (CB1R, $\mathrm{Cb1}^{-/}$mice) and in mice lacking $\mathrm{CB} 1 \mathrm{R}$ in the cerebellar parallel fibers, suggesting that CB1R downregulation in the cerebellar molecular layer plays a key role in THC-induced cerebellar deficits. Expression of CB2 cannabinoid receptor (CB2R) and $I l 1 b$ mRNA was increased under neuroinflammatory conditions in activated CD11b-positive microglial cells. Furthermore, administration of the immunosuppressant minocycline or an inhibitor of IL-1 $\beta$ receptor signaling prevented the deficits in cerebellar function in $\mathrm{Cb1}^{-/-}$and THC-withdrawn mice. Our results suggest that cerebellar microglial activation plays a crucial role in the cerebellar deficits induced by repeated cannabis exposure.
\end{abstract}

\section{Introduction}

Cannabis sativa preparations are the most consumed illicit drugs for recreational purposes (1). Delta9-tetrahydrocannabinol (THC) constitutes the main psychoactive component in those preparations (2). THC acts as a partial agonist of CB1 and CB2 cannabinoid receptors (CB1R and CB2R). CB1Rs are widely expressed in the brain where they control the release of neurotransmitters at the presynaptic level (3). In contrast, CB2Rs have been mainly localized in the immune system (4) and show a modest expression in neurons $(5)$ and microglia $(6,7)$ in the healthy brain.

Chronic THC exposure induces adaptive changes in the CNS typified by a downregulation of CB1R in animal models (8) and humans (9), but the effect of such exposure on CB2R is largely unknown. The marked decrease in the expression, density, and functional activity of CB1R after chronic THC exposure differ between brain areas (8). This downregulation is particularly relevant in the cerebellum, although it has been demonstrated that at least 3 days of daily CB1R agonist administration are required before reductions are detectable (10). In the cerebellum, CB1Rs are highly expressed in the molecular layer, specifically in the parallel fiber terminals on Purkinje cells (11), where they regulate long-term synaptic plasticity processes at these glutamatergic synapses (12). Indeed, long-term depression (LTD) at these synapses is thought to be predominant for cerebellar-dependent conditioned learning, specifically for delayed eyeblink conditioning (13). Thus, CB1Rs inhibit glutamate release in the cerebellar cortex (14), and the decrease in CB1R activity after subchronic cannabinoid exposure results in an enhancement of glutamatergic postsynaptic activity (14). Accordingly, repeated exposure to cannabis affects cerebellar associative learning in humans, as

Conflict of interest: The authors have declared that no conflict of interest exists. Citation for this article: J Clin Invest. 2013;123(7):2816-2831. doi:10.1172/JCI67569. detected in the delayed eyeblink conditioning paradigm (15), while forebrain function measured by the trace eyeblink conditioning paradigm is not affected (16). Similarly, constitutive KO mice lacking CB1R show a complete deficit in the acquisition of delayed eyeblink conditioning, but not in the trace eyeblink conditioning paradigm (17). Furthermore, when current and former cannabis users were compared in cerebellum-dependent delayed eyeblink conditioning, a disruptive effect in timing-related synaptic plasticity within the cerebellum persisted after the cessation of cannabis consumption (18). Altogether, cerebellar function is largely affected by CB1R deregulation and cannabis consumption (19).

Microglial cells are the main immune effector cells of the CNS and express low, if any, CB1R and CB2R in resting homeostatic conditions, while they express CB2R at detectable levels when microglia are activated (20). These cells are essential for normal functioning of the mature CNS, since they regulate innate immunity and provide extensive and continuous surveillance of parenchyma and synaptic contacts (21-23). In the healthy adult brain, resting microglial cells are characterized by a small soma with fine, highly ramified branches and low expression of surface antigens (24). Following brain stress or injury, local changes in the extracellular milieu promote a change in the microglial phenotype, referred to as microglial activation. Since microglial cells do not receive direct synaptic inputs, it has been proposed that neurotransmitter receptors expressed by microglia might induce and control this activation (25). For example, microglia express a variety of these receptors, including those for glutamate and GABA (26), that modulate their functional response (27). In this context, it is known that the main excitatory neurotransmitter, glutamate, acting on glutamate receptors expressed by microglial cells, plays a crucial role in classical microglial activation involving IL-1 $\beta$-enhanced expression $(26,28)$. This process includes mor- 

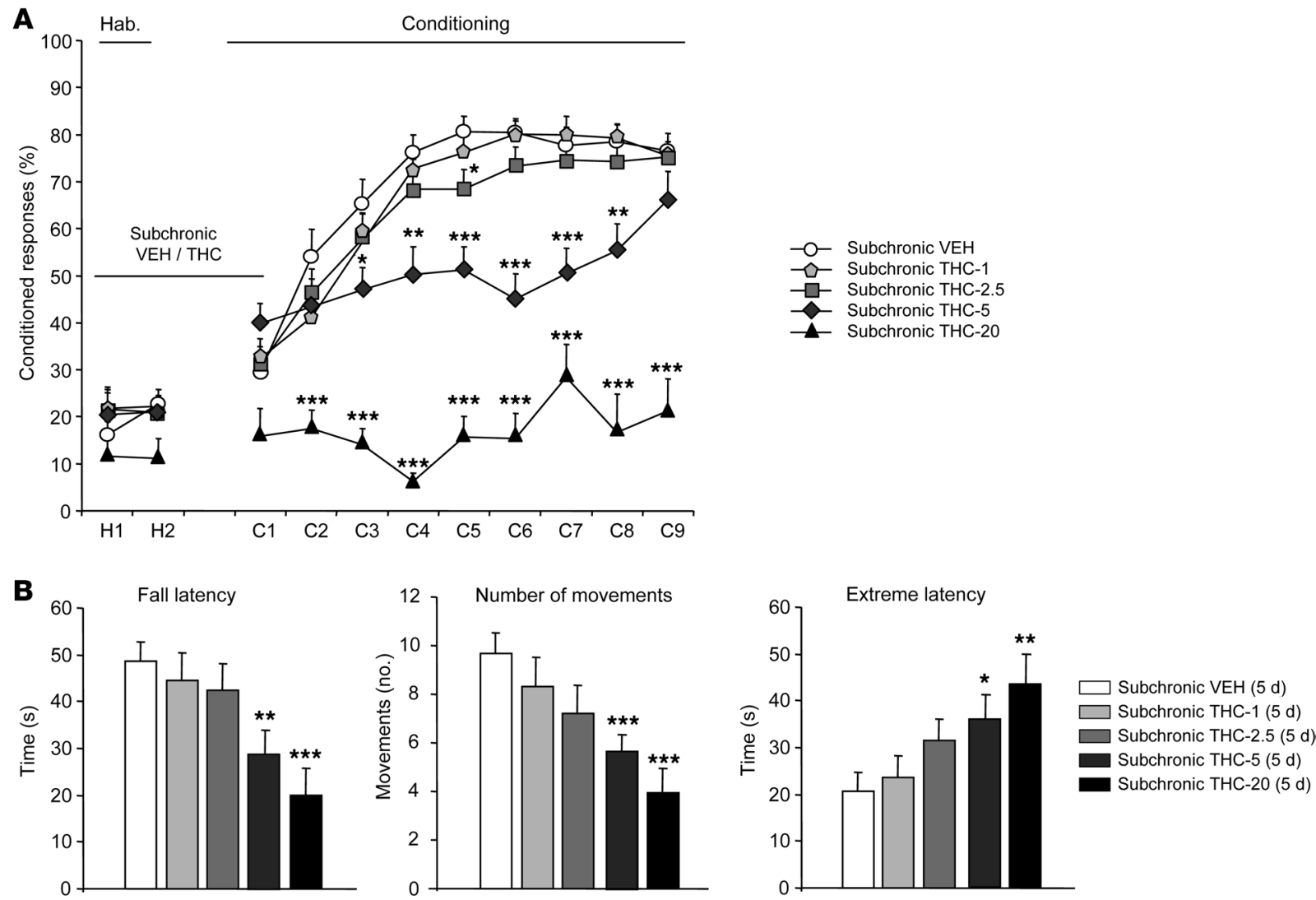

Figure 1

Cerebellar performance after subchronic THC exposure in mice. (A) Percentage of conditioned eyelid responses collected from mice that underwent subchronic THC $(1,2.5,5$, and $20 \mathrm{mg} / \mathrm{kg})$ or subchronic VEH $(n=9-12$ mice per group) treatment (see Supplemental Figure 1A for experimental chronogram). (B) Motor coordination analysis using the coat-hanger test after subchronic exposure to THC (1, 2.5, 5, and $20 \mathrm{mg} / \mathrm{kg})$ or subchronic VEH conditions 5 days after spontaneous withdrawal $(n=11-17$ mice per group). Impaired motor coordination was revealed by fall latency, number of movements to reach the end of the coat hanger, and extreme latency. ${ }^{\star} P<0.05 ;{ }^{* \star} P<0.01 ;{ }^{* \star *} P<0.001$ versus subchronic VEH treatment. Hab., habituation.

phological changes from the resting phenotype to an amoeboid morphology accompanied by changes in the expression of proinflammatory and antiinflammatory genes $(29,30)$.

In this study, we report that subchronic THC exposure activates cerebellar microglia. This activation, revealed by changes in the microglial phenotype and by enhanced CD $11 b$ and IL- $1 \beta$ expression, correlates with the downregulation of cerebellar CB1R in the molecular layer. The localized neuroinflammatory status described herein has profound relevance in cerebellar-dependent conditioned learning and motor coordination performance. These data suggest that the cerebellar neuronal/glial circuitry is controlled by CB1Rs in the molecular layer of the cerebellum, and the impairment of this mechanism leads to functional alteration of this area of the brain. Considering that the neuronal circuits involved in this response are similar in mice and humans, we hypothesize that the mechanisms described herein would be directly involved in the cerebellar deficits recently reported in chronic cannabis users.

\section{Results}

Subchronic THC results in cerebellar conditioned learning deficits and motor coordination alteration. We followed a short schedule of systemic THC administration known to produce cannabinoid phys- ical dependence (31) and examined whether cerebellar function would be compromised after THC exposure. Mice received THC $(1,2.5,5$, or $20 \mathrm{mg} / \mathrm{kg}$, i.p.) twice daily over a 5 -day period and once on the sixth day. We used the delayed eyeblink conditioning paradigm, since the cerebellum contributes critically to the acquisition (32) and performance (33) of this task. This learning model involves the same neuronal circuits in rodents and humans (34) and is specifically affected in current and former cannabis smokers $(15,18)$. The first conditioning session was performed on the last day of THC (or vehicle) treatment starting 4 hours after the last drug administration (Supplemental Figure 1A; supplemental material available online with this article; doi:10.1172/ JCI67569DS1). The percentage of conditioned responses was measured every day for 9 days. Mice receiving subchronic THC at a dose of $1,2.5,5$, or $20 \mathrm{mg} / \mathrm{kg}$ showed a dose-dependent deficit in cerebellar associative learning (Figure 1A). Specifically, this deficit was sustained from the third to the eighth day after treatment cessation in the $5 \mathrm{mg} / \mathrm{kg}$ (THC-5) or $20 \mathrm{mg} / \mathrm{kg}$ (THC-20) groups, while this was only revealed on the fifth day in the THC-2.5 group (Figure 1A). In contrast, the conditioned learning performance of the THC-1 group was similar to that of the vehicle group (Figure 1A). Considering these results, we studied the motor coordination 

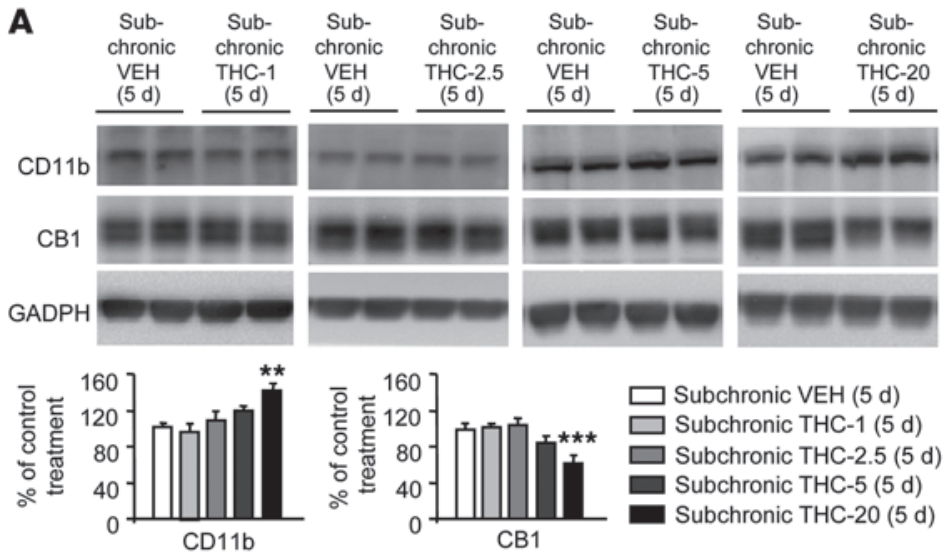

B
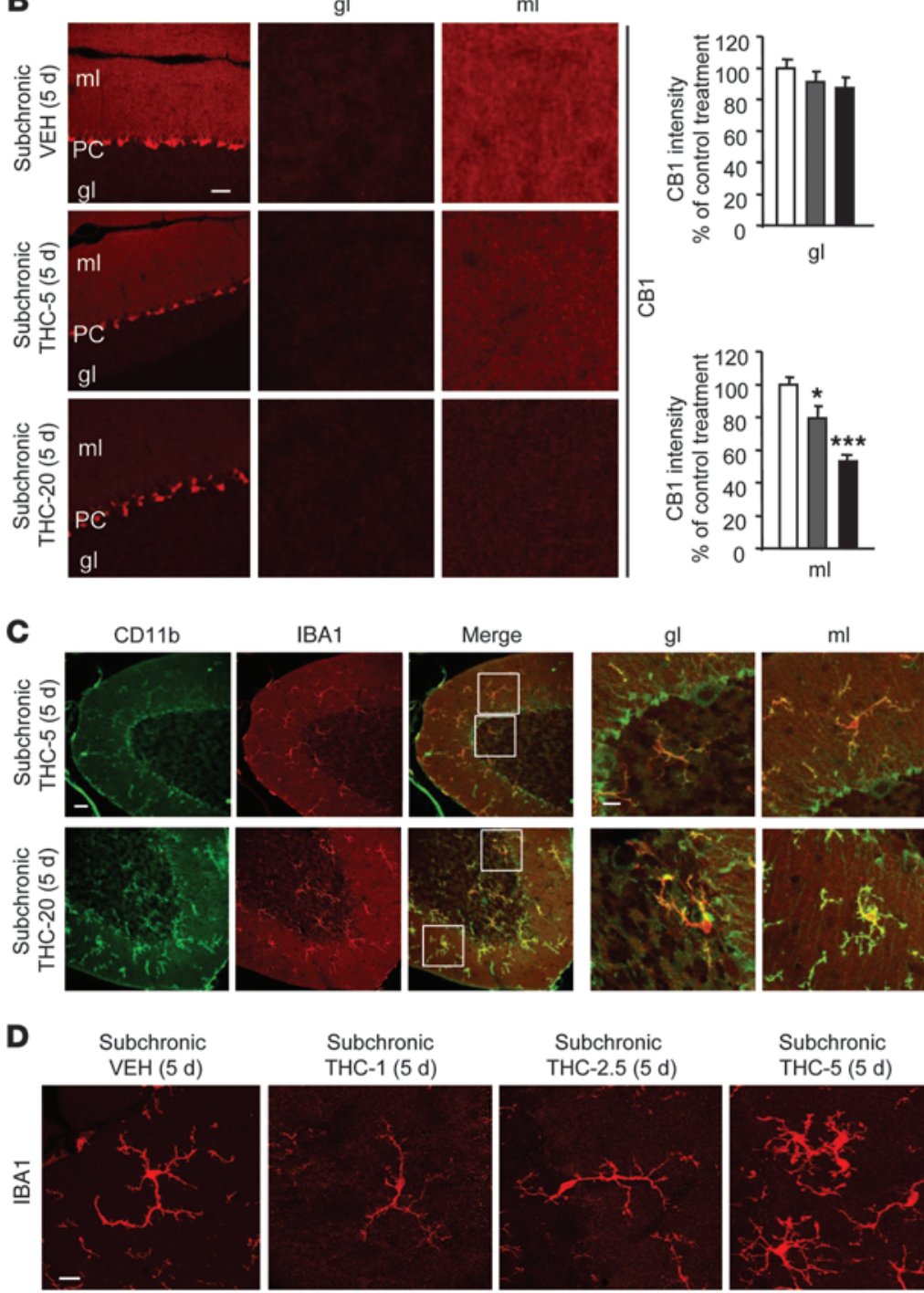

Subchronic THC-1 (5 d)
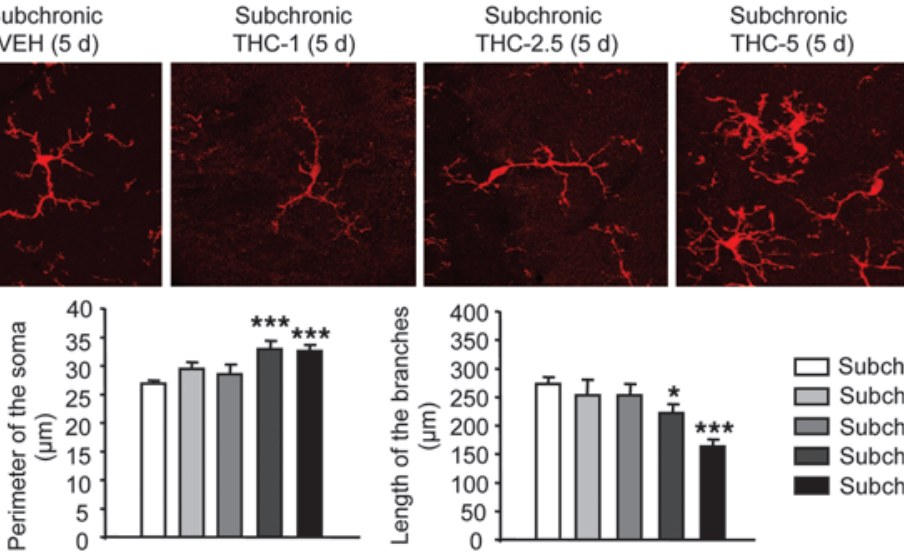

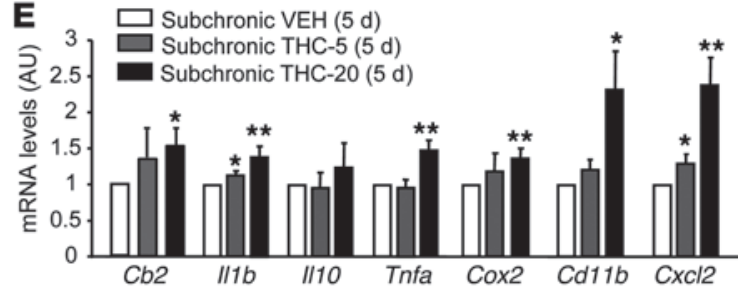

F
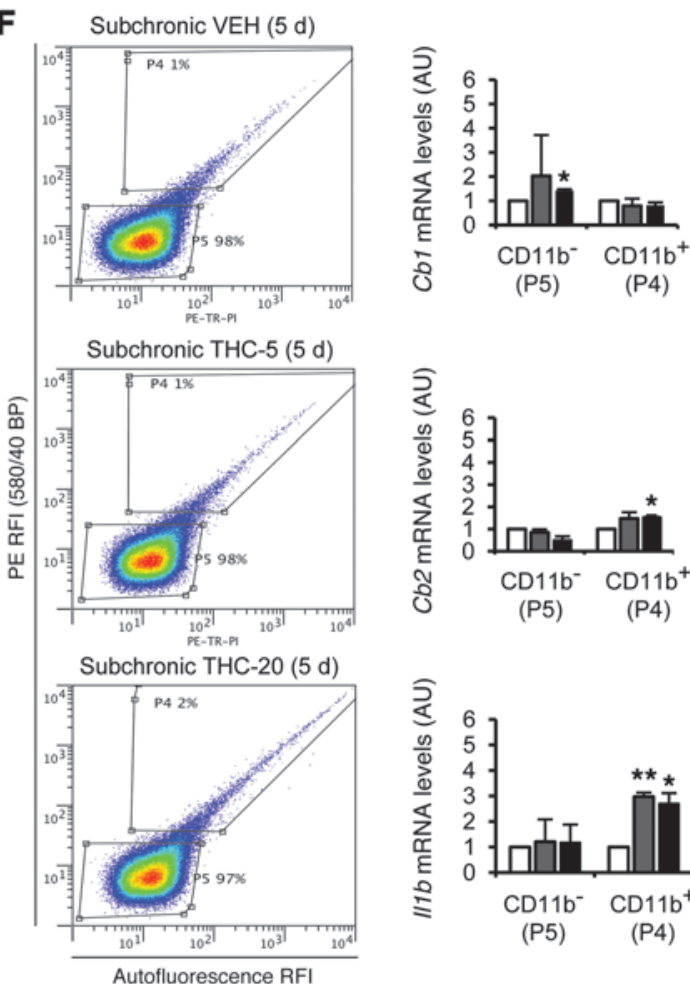

(610/20 BP)
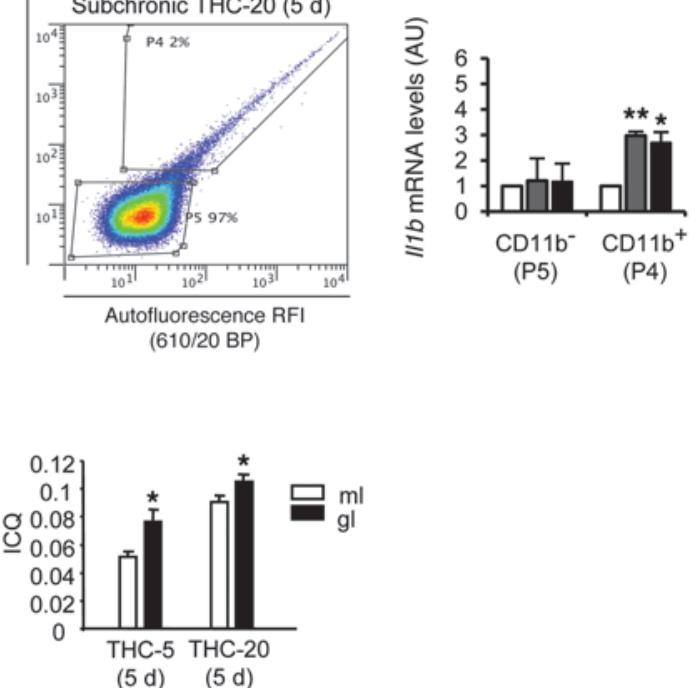

Subchronic THC-20 (5 d)

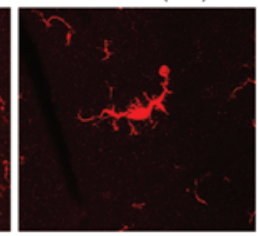

$\square$ Subchronic VEH (5 d)

$\square$ Subchronic THC-1 (5 d)

$\square$ Subchronic THC-2.5 (5 d)

Subchronic THC-5 (5 d)

Subchronic THC-20 (5 d) 


\section{Figure 2}

Cerebellar responses 5 days after THC treatment cessation. (A) Immunoblot and quantification of CD11b and CB1R in cerebellar homogenates from mice processed 5 days after subchronic treatment ( $n=5-6$ mice per group). The optical density of CD11b and CB1R was normalized to GAPDH in the same samples. (B) Immunofluorescence and quantification of CB1R intensity in the granular and molecular layers of the cerebellum from mice processed 5 days after subchronic treatment ( $n=3$ mice per group, 5 images per mouse). Scale bar: $75 \mu \mathrm{m}$. (C) Immunolocalization and ICQ of IBA1 (red) and CD11b (green) in the molecular and granular layers of the cerebellum after treatment ( $n=3$ mice per group, 3 images per mouse). Scale bars: $100 \mu \mathrm{m}$. (D) Morphological analysis of IBA1+ microglial cells in the molecular layer of the cerebellum ( $n=4$ mice per group, 4 cells per mouse). See Supplemental Figure 17 for details. Scale bar: $25 \mu \mathrm{m}$. (E) Analysis of $\mathrm{Cb} 2 r$ mRNA expression and inflammation-related genes in cerebellar samples ( $n=7-8$ mice per group). (F) Flow cytometric analysis of CD11b expression and qRT-PCR analysis of acutely dissociated cerebellar cells from VEH-, THC-5-, and THC-20-treated mice ( $n=3$ per group). Sorted CD11 b+ population (P4) and CD11b- population (P5) in THC-treated mice showed a differential expression of $C b 1 r, C b 2 r$, and $/ l 1 b$. ${ }^{\star} P<0.05$; ${ }^{* \star} P<0.01 ;{ }^{* \star} P<0.001$ versus subchronic VEH plus SAL ( 5 days).

skills on the fifth day after THC cessation in a different set of mice using the accelerating rotarod (Supplemental Figure 2) and coathanger tests (Figure 1B). Accelerating rotarod analysis showed learning impairment in this coordination task in THC-withdrawn mice compared with the control group at the same doses revealed in the conditioning paradigm (5 and $20 \mathrm{mg} / \mathrm{kg}$ ) (Supplemental Figure 2). More robust results were observed using the coat-hanger test (Figure 1B), a more demanding test that allows for the detection of fine alterations in motor coordination function. Five days after cessation of subchronic THC treatment, the mice showed a dose-dependent alteration in several coordination parameters (fall latency, number of movements along the hanger, and extreme latency) compared with the vehicle-treated mice. We observed significant effects at the same THC doses (5 and $20 \mathrm{mg} /$ $\mathrm{kg}$ ) as in the previous paradigms (Figure 1B). However, no significant differences between groups were observed when equilibrium was assessed with the rod test (Supplemental Figure 3). Together, these data reveal the cerebellar deficits associated with THC exposure and subsequent withdrawal.

THC withdrawal produces molecular and cellular signs of cerebellar neuroinflammation. Five days after THC treatment cessation, cerebellar homogenates were analyzed by Western blotting and showed a THC dose-dependent increase in expression of the microglial activation marker CD11b and a decreased expression of CB1R (Figure 2A). As revealed by immunofluorescence staining and CB1R intensity analysis, the decrease in CB1R expression occurred mainly in the cerebellar molecular layer of THC-withdrawn mice (5 and $20 \mathrm{mg} / \mathrm{kg}$ ) (Figure 2B) In addition, double immunostaining and colocalization analysis of IBA1 and CD11b on cerebellar slices under similar conditions demonstrated that microglial activation took place mainly in the molecular layer of the cerebellum, rather than in the granular layer (Figure 2C). In this cerebellar layer, microglia acquired a bushy morphology according to the perimeter of the soma and the length of the branches after THC treatment cessation (Figure 2D). Notably, the microglial activation phenotype was not detectable in other brain areas where CB1Rs are heavily expressed such as the hippocampus, frontal cortex, or striatum (Supplemental Figure 4).
Quantitative analysis of mRNA for $C b 2 r$ and the neuroinflammatory markers Il1b, Tnfa, Cox2, Cd11b, and Cxcl2 indicated that the expression of these genes is enhanced in the cerebellum 5 days after the cessation of subchronic THC treatment at doses of 5 and $20 \mathrm{mg} / \mathrm{kg}$ (Figure 2E).

In an additional experimental group, the cannabinoid withdrawal syndrome was precipitated after subchronic THC $(20 \mathrm{mg} / \mathrm{kg})$ treatment by rimonabant $(10 \mathrm{mg} / \mathrm{kg}$, i.p.) administered 4 hours after the last THC injection (31). Control groups that were subchronically treated with vehicle and that were receiving an acute challenge of rimonabant or its vehicle were also run in parallel. We found that under rimonabant-triggered cannabinoid withdrawal, the results were similar in terms of the reactivity of cerebellar microglia (Supplemental Figure 5, A and B) and the downregulation of cerebellar CB1R (Supplemental Figure 5C) to those reported here after spontaneous cessation of subchronic THC treatment.

We hypothesized that the neuroinflammatory phenotype observed in the cerebellar molecular layer 5 days after THC cessation would respond to a possible restricted alteration in the extracellular milieu resulting from deregulated glutamate handling in the parallel fiber terminals as a consequence of CB1R downregulation. As expected, a strong decrease in CB1R expression was also detected in the cerebellum at the end of subchronic THC treatment (Supplemental Figure 6A), in agreement with previous studies (8). In contrast, no changes in CD11b expression were detected in the cerebellar homogenates (Supplemental Figure 6A), although the microglial morphology was slightly altered (Supplemental Figure 6B). Fluoro-Jade B assay revealed no signs of cellular death in the cerebellum at the end of subchronic THC treatment, suggesting that the microglial reactivity was not associated with cytotoxicity processes (Supplemental Figure 7, A and B). Moreover, we observed no signs of microglial (Supplemental Figure 7, C and D) or astroglial (Supplemental Figure 7, E and F) proliferation in the cerebellum at the end of this treatment, as measured by cell counting and immunoblot analysis of IBA1 and glial fibrillary acidic protein (GFAP) expression. Similarly, the neuroinflammatory markers $I l 1 b$, Tnfa, Cox2, Cxcl2, and Il10 were not altered in the cerebellum at the end of THC treatment (Supplemental Figure 7G).

When the time course of these effects mediated by THC exposure was studied, we observed that $\mathrm{CD} 11 \mathrm{~b}$ expression increased in the cerebellum only 5 days after THC cessation, while the downregulation of CB1R expression reached its maximum level at the end of subchronic THC treatment, slowly recovering afterward (Supplemental Figure 8A). The time course for the effect of THC exposure on microglial morphology revealed a progressive modification of these cells to the activated phenotype (Supplemental Figure 8B). In addition, we sorted the acutely dissociated cerebellar cells, positive $\left(\mathrm{CD} 11 \mathrm{~b}^{+}\right.$, population 4, $\mathrm{P} 4$ ) or negative (CD11b $\mathrm{b}^{-}$, population 5, $\mathrm{P} 5$ ) for $\mathrm{CD} 11 \mathrm{~b}$, from mice receiving subchronic vehicle, THC-5, or THC-20 and sacrificed 5 days after treatment cessation. These cells were analyzed by RT-PCR for mRNA expression of Cb1r, Cb2r and the proinflammatory cytokine $I l 1 B$ (Figure $2 \mathrm{~F}$ ). Under these conditions, the percentage of $\mathrm{CD} 11 \mathrm{~b}^{+}(\mathrm{P} 4)$ cells slightly increased in the THC-20 group. In the $\mathrm{CD}_{11 \mathrm{~b}^{+}}(\mathrm{P} 4)$ population, mainly corresponding to cells positive for IBA1 staining (Supplemental Figure 9), enhanced expression of $C b 2 r$ and $I l 1 b$ was observed (Figure 2F). However, CD11b- (P5) cells did not reveal modulated $C b 2 r$ or $I l 1 b$ gene expression, but rather showed enhanced expression of Cb1r mRNA (Figure 2F). 
A
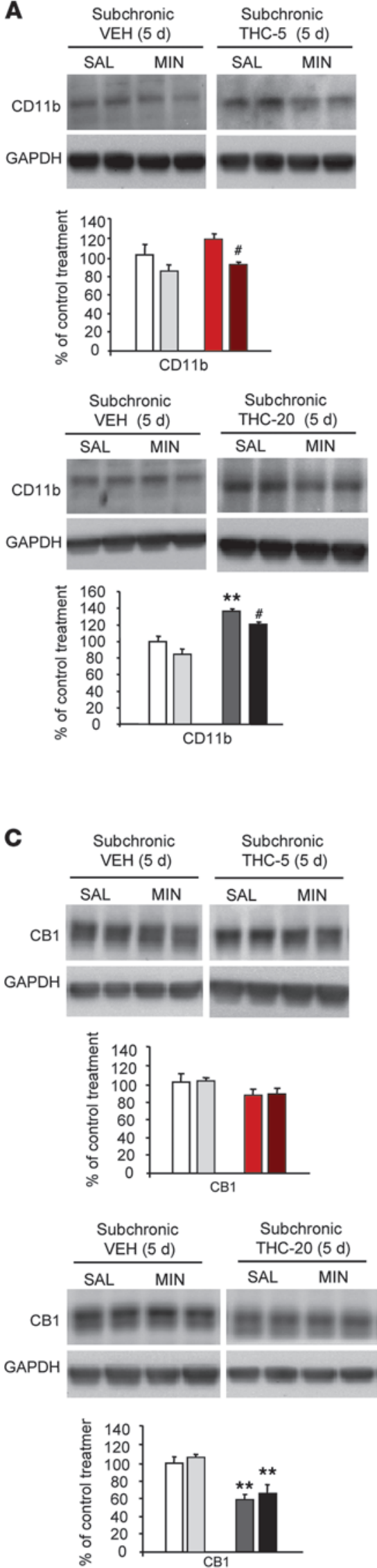

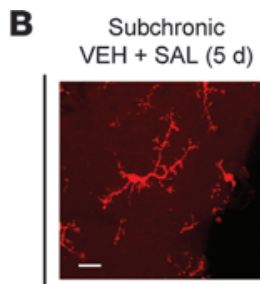

Subchronic THC-5 + SAL (5

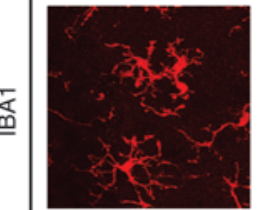

Subchronic THC-20 + SAL (5 d) THC-20 + MIN
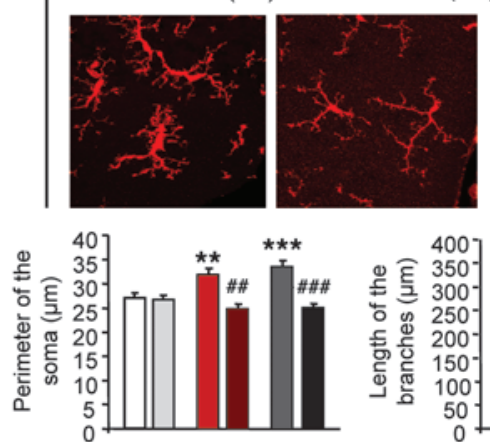

$\square$ Subchronic VEH + SAL (5 d) Subchronic THC-5 + SAL (5 d)

$\square$ Subchronic THC-20 + SAL (5 d)

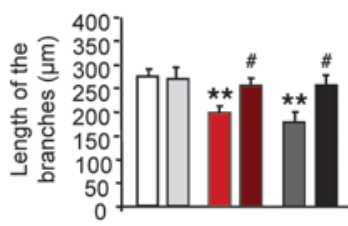

\section{0}

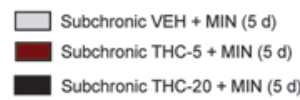

D Subchronic
$V E H+S A L(5$ Subchronic VEH + MIN (5 d)
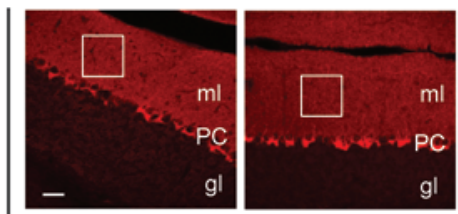

Subchronic

Subchronic THC-5 + SAL (5 d) THC-5 + MIN (5 d)

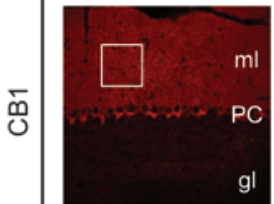

Subchronic

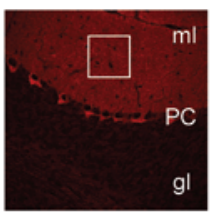

Subchronic THC-20 + SAL (5 d) THC-20 + MIN (5 d)
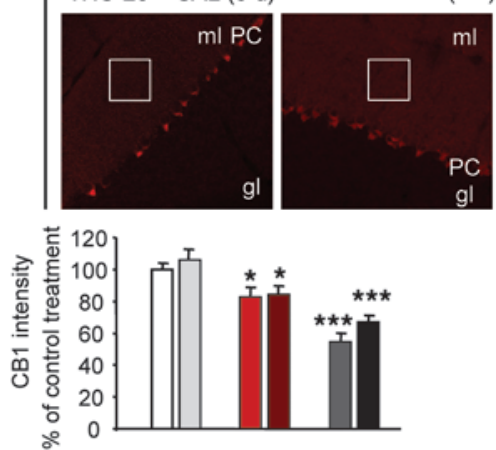

Figure 3

MIN administration after subchronic THC exposure prevents the activation of microglia in the cerebellum. (A) Immunoblot detection and quantification of cerebellar CD11b ( $n=5$ mice per group) at the end of subchronic exposure to MIN or SAL under subchronic THC (5 and $20 \mathrm{mg} / \mathrm{kg}$ ) and subchronic VEH treatment conditions. (B) Morphological analysis of IBA1+ cells in the cerebellar cortex ( $n=3-4$ mice per group, 4-5 cells per mouse). Scale bar: $25 \mu \mathrm{m}$. (C) Immunoblot detection and quantification of cerebellar CB1R $(n=6$ mice per group) at the end of MIN or SAL exposure under subchronic THC (5 and $20 \mathrm{mg} / \mathrm{kg}$ ) and subchronic VEH treatment conditions. (D) Immunolocalization and quantification of CB1R intensity in the cerebellar molecular layer at the end of subchronic exposure to MIN or SAL under subchronic THC (5 and $20 \mathrm{mg} / \mathrm{kg}$ ) and subchronic VEH treatment conditions ( $n=4$ mice per group; 3-4 images per mouse). Note the downregulation of $\mathrm{CB} 1 \mathrm{R}$ in the molecular layer of the cerebellum 5 days after the end of THC- 5 and THC20 subchronic treatments. Scale bar: $75 \mu \mathrm{m} .{ }^{*} P<0.05$; ${ }^{\star \star} P<0.01 ;{ }^{* *} P<0.001$ versus subchronic VEH plus SAL (5 days); $\#<<0.05$; \#\# $P<0.01$; \#\#\# $P<0.001$ versus subchronic THC (5 or $20 \mathrm{mg} / \mathrm{kg}$ ) plus SAL (5 days) treatment. 
A

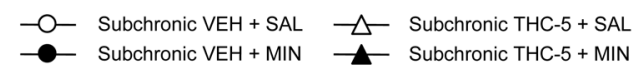

Hab.

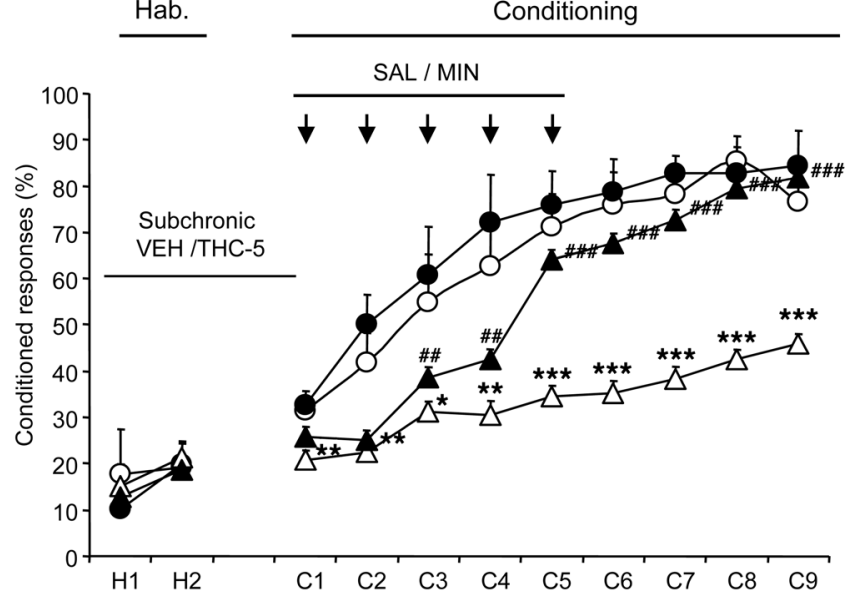

B

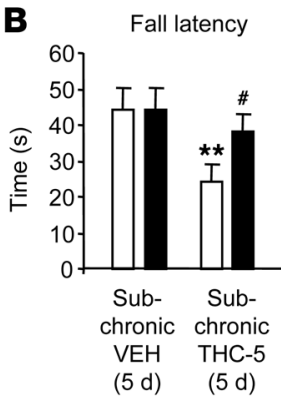

No. of movements

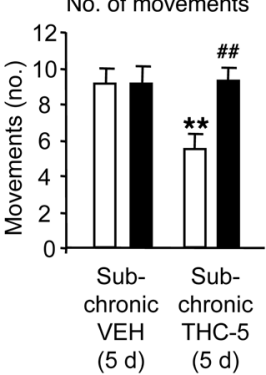

Extreme latency

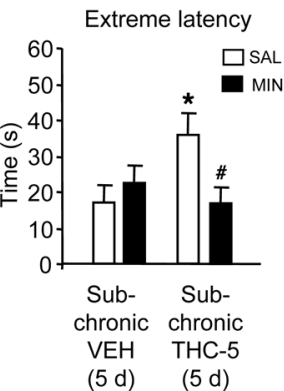

C

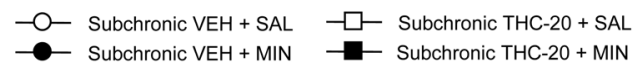

Hab.

Conditioning

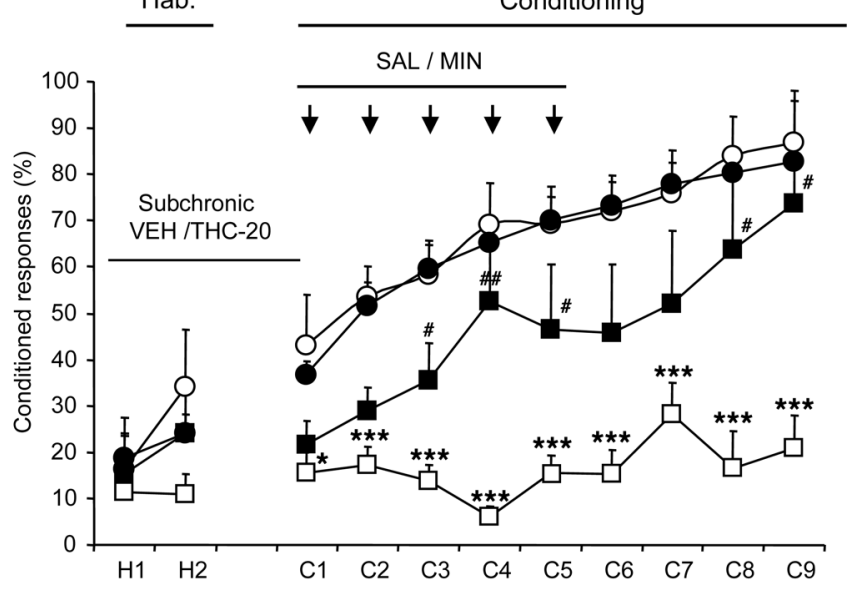

D

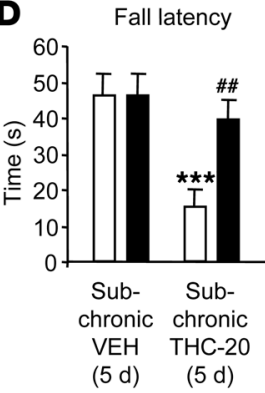

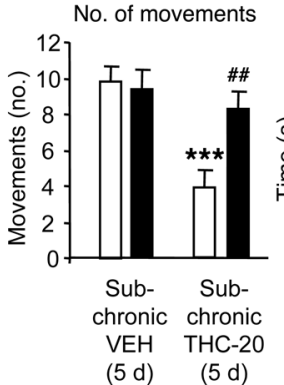

Extreme latency

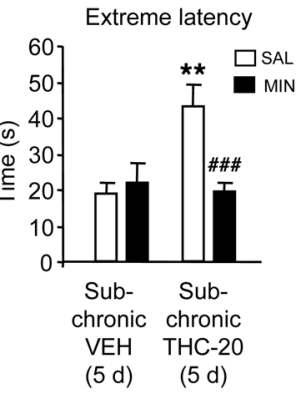

Figure 4

MIN treatment prevents the cerebellar deficits produced by THC exposure. (A) Percentage of conditioned eyelid responses collected from mice receiving MIN or SAL after subchronic THC-5 or VEH treatment $(n=9-11$ mice per group) (see Supplemental Figure 1B for experimental chronogram). (B) Motor coordination analysis at the end of subchronic exposure to MIN or SAL in mice that had previously received THC-5 or VEH ( $n=15$ mice per group). Alterations in motor coordination evaluated in the coat-hanger test were ameliorated by subchronic MIN administration. (C) Percentage of conditioned eyelid responses collected from mice receiving MIN or SAL after subchronic THC-20 or VEH treatment $(n=9-11$ mice per group). See Supplemental Figure 1B for experimental chronogram. (D) Motor coordination analysis at the end of subchronic exposure to MIN or SAL in mice that had previously received THC-20 or VEH ( $n=15$ mice per group). Impairment in motor coordination skills measured by the coat-hanger test was prevented with subchronic MIN treatment. ${ }^{*} P<0.05$; ${ }^{* *} P<0.01 ;{ }^{* *} P<0.001$ versus subchronic VEH plus SAL (5 days) treatment. ${ }^{~} P<0.05$; $\# P<0.01$; \#\#\# $P 0.001$ versus subchronic THC (5 or $20 \mathrm{mg} / \mathrm{kg}$ ) plus SAL (5 days) treatment.

Therefore, the inflammatory phenotype in the cerebellum was progressively enhanced after subchronic THC cessation and correlated with the poor cerebellar functioning.

Blockade of microglial activation ameliorates cerebellar deficits. We used the tetracycline antibiotic minocycline, an immunosuppressant with inhibitory effects on microglial activation (35), to evaluate the role of this process in the motor coordination deficit described above after cessation of the subchronic THC treatment. Minocycline (40 $\mathrm{mg} / \mathrm{kg}$, i.p.) was administered 1 hour after the last THC injection (5 or $20 \mathrm{mg} / \mathrm{kg}$, i.p.) on the sixth day of THC treatment, and once a day for 5 days. Subchronic minocycline treatment reversed the enhancement of CD11b levels in the cerebellum that had been promoted by subchronic THC administration (Figure 3A). Moreover, minocycline treatment normalized microglial morphology to control levels with regard to both the perimeter of the soma and the length of the branches (Figure 3B). Cerebellar CB1R expression was not affected after minocycline administration, as detected by immunoblotting of cerebellar samples (Figure 3C) and by immunofluorescence detection in the molecular layer of the cerebellum (Figure 3D).
The compromised cerebellar conditioned learning of the THC-withdrawn mice was normalized after minocycline blockade of microglial activation. As described above, the first conditioning session (C1) was performed on the last day of subchronic THC (5 or $20 \mathrm{mg} / \mathrm{kg}$ ) or vehicle treatment, which coincided with the first day of minocycline (or saline) administration (C1) (Figure 4, A and C, and Supplemental Figure 1B). As shown previously, the groups that had received subchronic THC showed a dose-dependent deficit in associative learning compared with the control groups during the conditioning phase (Figure 4, A and C). The administration of minocycline increased the percentage of conditioned responses starting on the third day of treatment (C3) (Figure 4, A and C). In another experimental set, THC-withdrawn mice were tested for motor coordination on the fifth and last day of minocycline administration using the coat-hanger test. Minocycline also reversed the motor coordination impairment induced by THC, improving coat-hanger test performance compared with that of the subchronically THC-treated mice receiving saline (Figure 4, 
A
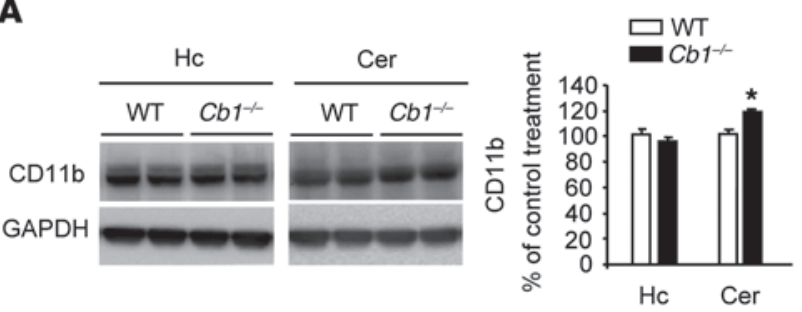

C
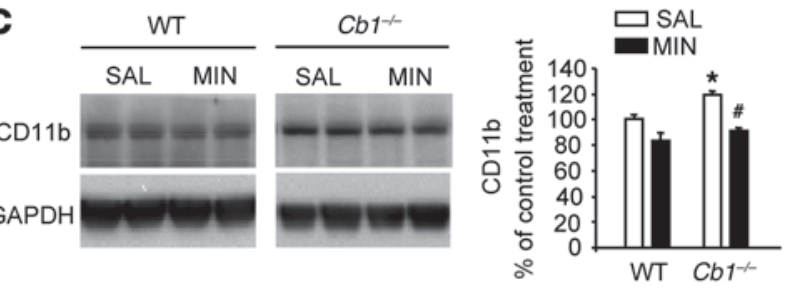

B
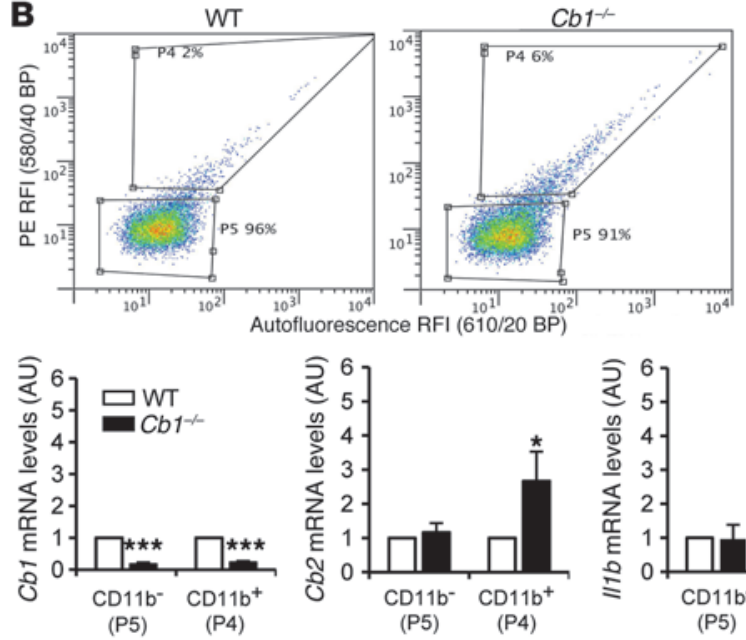
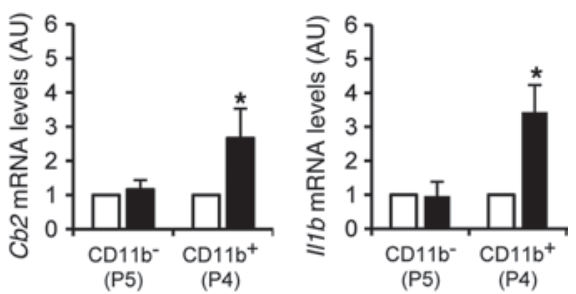

D
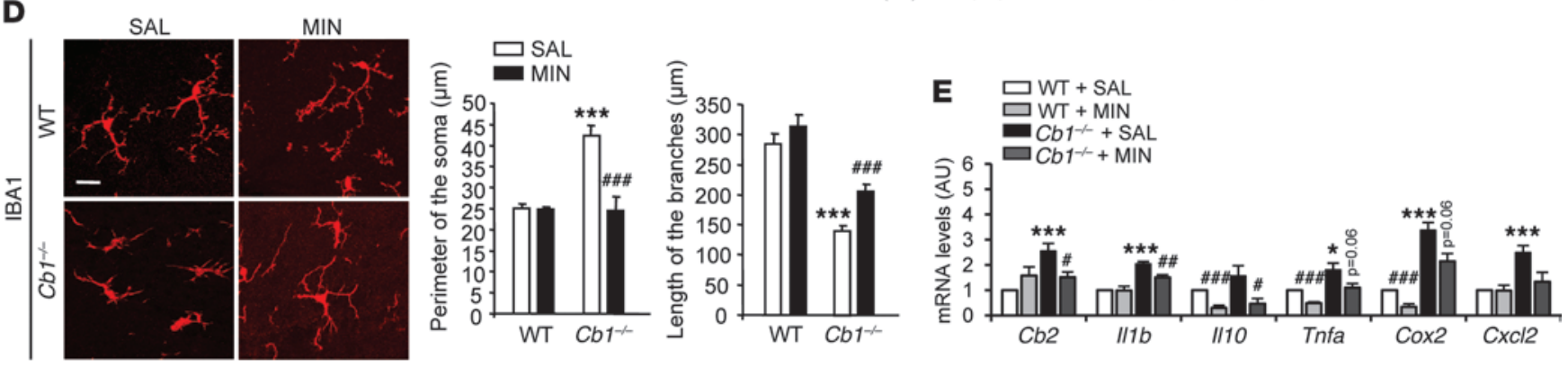

F

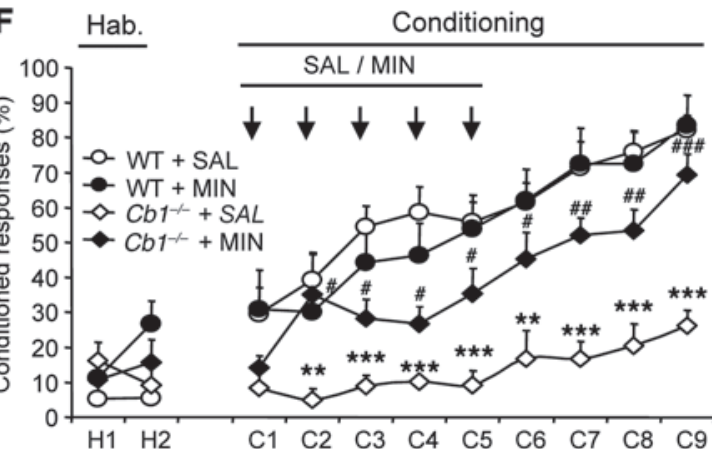

G
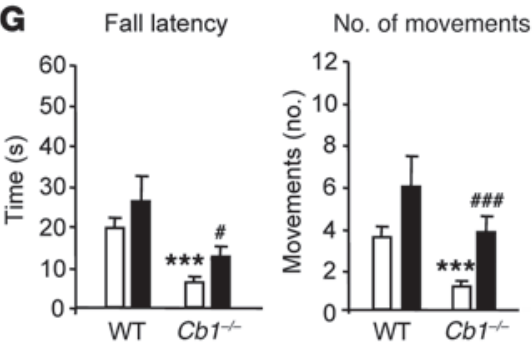

Extreme latency

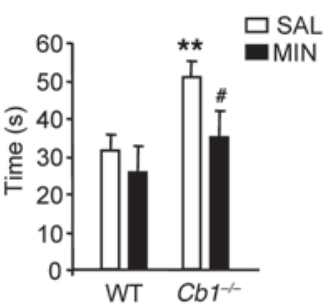

\section{Figure 5}

Genetically downregulated CB1R promotes cerebellar neuroinflammation and reversible cerebellar deficits. (A) CD11b detection in WT and Cb1r KO $\left(\mathrm{Cb}^{-1-}\right)$ mice ( $n=5$ per group). CD11b quantification was normalized to GAPDH. ${ }^{*} P<0.05$ versus WT. (B) Flow cytometric analysis of CD11b expression and quantitative RT-PCR (qRT-PCR) analysis of acutely dissociated cerebellar cells from WT and Cb1-/- mice $(n=3-4$ per group). (C) CD11b in cerebellar homogenates in WT and $C b 1^{-/-}$mice after treatment ( $n=6$ mice per group). CD11b detection was normalized to GAPDH. ${ }^{*} P<0.05$ versus WT plus SAL; ${ }^{\#} P<0.05$ versus $C b 1^{-1-p l u s ~ S A L . ~(D) ~ M o r p h o l o g i c a l ~ a n a l y s i s ~ o f ~ I B A ~} 1^{+}$cells in the cerebellar cortex ( $n=3-5$ mice per group, 5 cells per mouse) of WT and Cb1-- mice after treatment. Scale bar: $25 \mu \mathrm{m}$. ${ }^{* \star \star} P<0.001$ versus WT plus SAL; $\# \# \#<0.001$ versus $\mathrm{Cb}^{-1-}$ plus SAL. (E) Analysis of $\mathrm{Cb} 2 \mathrm{r}$ mRNA expression by qRT-PCR and inflammation-related genes in the cerebellum ( $n=5-6$ per group). ${ }^{*} P<0.05,{ }^{* *} P<0.01,{ }^{* *} P<0.001$ versus WT. $(\mathbf{F})$ Percentage of conditioned eyelid responses collected from WT and $C b 1^{-/-}$ mice treated with MIN or SAL ( $n=7-10$ mice per group). See Supplemental Figure 12A for experimental chronogram. ${ }^{* *} P<0.01 ;{ }^{* * *} P<0.001$ versus WT plus SAL; ${ }^{P} P<0.05$, \#\# $P<0.01$, \#\#\#\# $P<0.001$ versus $C b 1^{-/-}$plus SAL. (G) Motor coordination analysis in WT and $C b 1^{-/-}$mice after

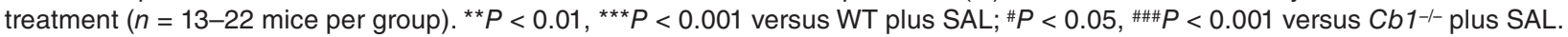

B and D). Therefore, microglial activation blockade correlates with a reduction in the motor coordination deficits observed in spontaneous THC-withdrawn mice. In an additional experiment, we verified that acute minocycline administration did not modify the somatic manifestations of rimonabant-mediated $(10 \mathrm{mg} / \mathrm{kg}$, i.p.) withdrawal syndrome after subchronic THC (20 mg/kg, i.p.) treatment, which discards a possible acute effect of minocycline on the manifestation of withdrawal symptoms (Supplemental Figure 10).

Together, these results suggest the involvement of microglial activation on cerebellar conditioned learning and motor coordination impairments promoted by subchronic THC administration, given that optimal performance on both functional tests requires intact cerebellar-mediated functioning. 
A

A Hab

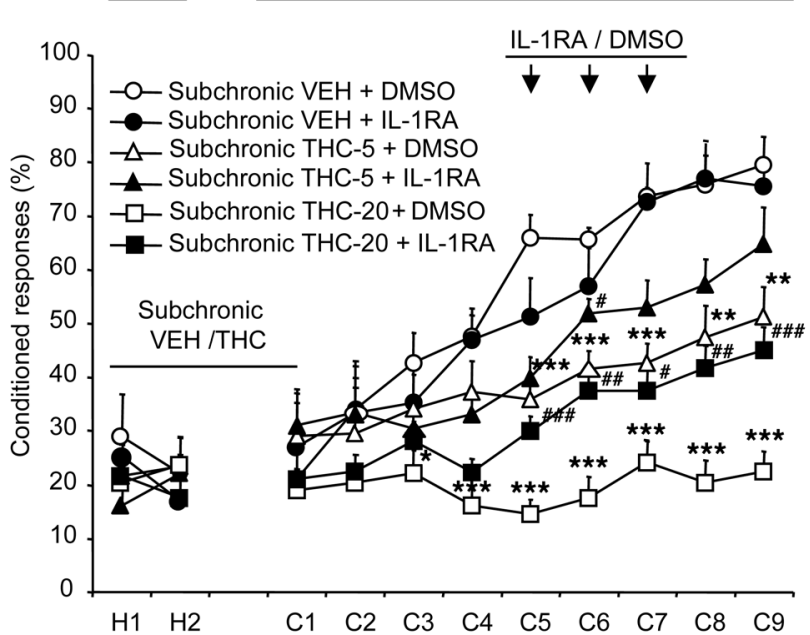

B

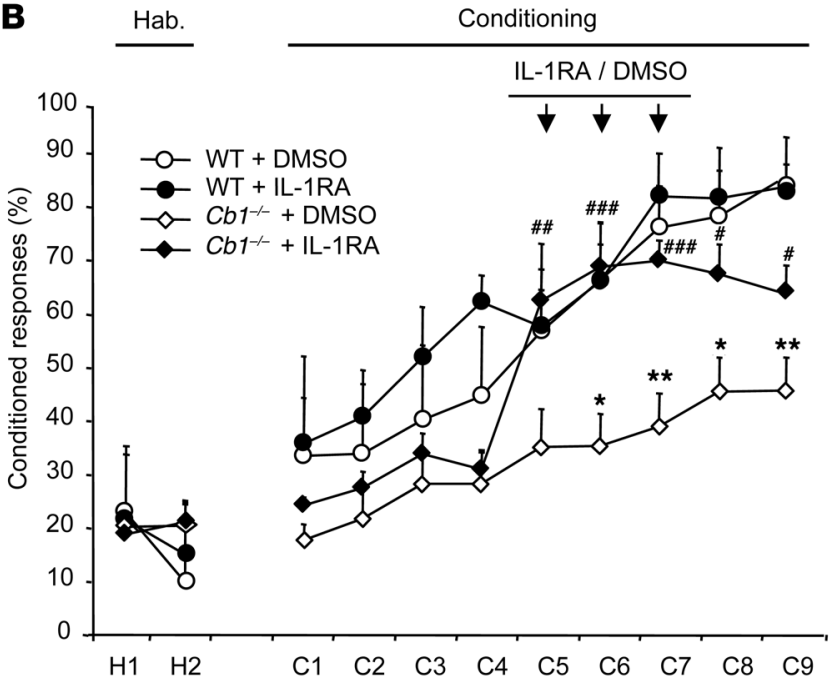

C

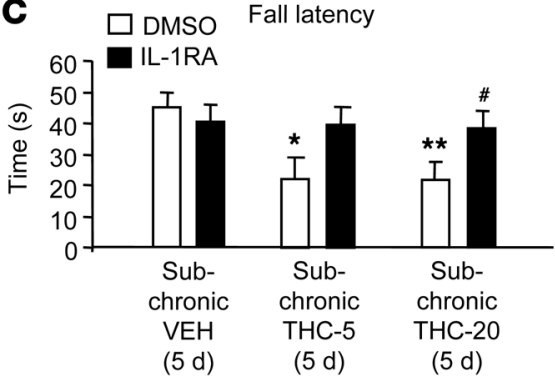

D

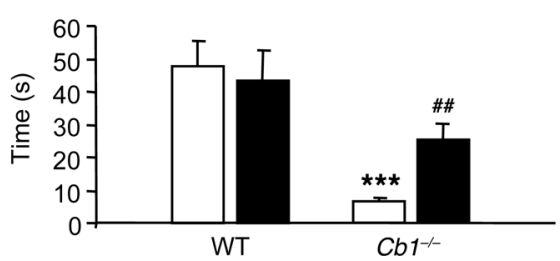

No. of movements

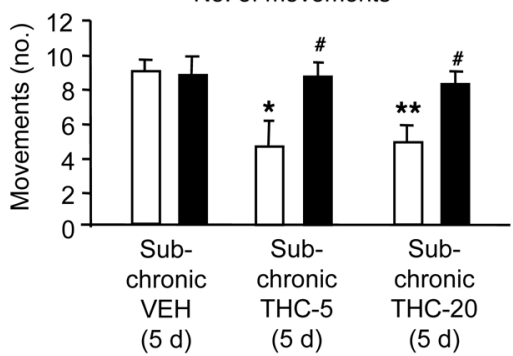

No. of movements

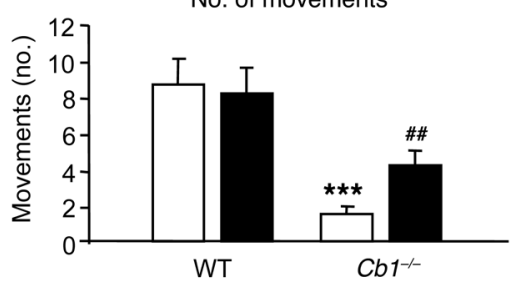

Extreme latency

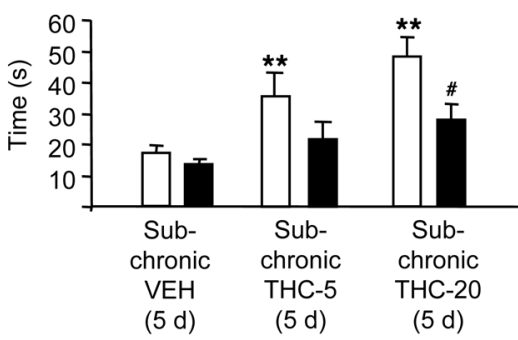

Extreme latency

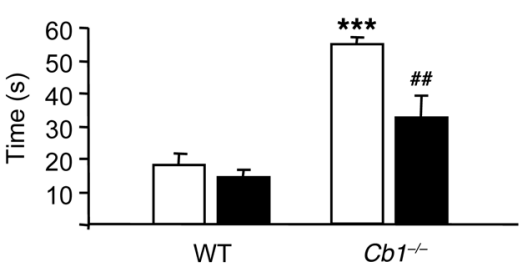

Figure 6

Acute IL-1RA administration resolves the cerebellar deficit resulting from cannabinoid receptor deregulation and neuroinflammation. (A) Percentage of conditioned eyelid responses collected from mice subchronically treated with THC ( 5 and $20 \mathrm{mg} / \mathrm{kg}$ ) and VEH ( $n=5-10 \mathrm{mice}$ per group). See Supplemental Figure 1C for experimental chronogram. ${ }^{\star} P<0.05,{ }^{\star \star} P<0.01,{ }^{\star \star *} P<0.001$ versus subchronic VEH treatment plus DMSO; ${ }^{\#} P<0.05$, ${ }^{\#} P<0.01$, \#\#\# $P<0.001$ versus subchronic THC (5 or $20 \mathrm{mg} / \mathrm{kg}$ ) treatment plus DMSO. (B) Percentage of conditioned eyelid responses collected from WT and $\mathrm{Cb}^{-1-}$ mice $\left(n=5-10\right.$ per group). See Supplemental Figure 12B for experimental chronogram. ${ }^{*} P<0.05$, ${ }^{* \star} P<0.01$ versus WT plus DMSO; $\# P<0.05$, \#\# $P<0.01$, \#\#\# $P<0.001$ versus $C b 1^{-1-}$ plus DMSO. (C) Motor coordination analysis with the coat-hanger test in subchronic THC ( 5 and $20 \mathrm{mg} / \mathrm{kg}$ ) and subchronic VEH conditions 5 days after spontaneous withdrawal $(n=9-15$ mice per group). Four hours before the test, mice received an acute injection of IL-1RA (100 mg/kg, i.p.) or its VEH (DMSO). ${ }^{*} P<0.05,{ }^{* *} P<0.01$ versus subchronic VEH. (D) Motor coordination analysis with the coat-hanger test in WT and $\mathrm{Cb}^{-1-}$ mice $(n=8-13$ per group). Four hours before the test, mice received an injection of IL-1RA or its VEH. ${ }^{* \star} P<0.001$ versus WT plus DMSO; ${ }^{\# \# P}<0.01$ versus $\mathrm{Cb} 1^{-/-}$plus DMSO.

CB1R downregulation is critical for cerebellar neuroinflammation and function. To evaluate the relevance of CB1R downregulation in the neuroinflammatory and behavioral alterations reported here, we investigated these responses in $\mathrm{CB} 1 \mathrm{R} \mathrm{KO}$ mice $\left(\mathrm{Cb}^{-/-}\right)$. Mild motor coordination deficits have been previously reported in Cb1 ${ }^{-/-}$adult mice (3-5 months of age), as demonstrated by rotarod (36) and eyeblink conditioning tests (17). We observed increased CD11b expression in the cerebellum, but not in the hippocampus of naive $\mathrm{Cb}^{-/-}$mice (Figure 5A). Moreover, the percentage of acutely dissociated cerebellar cells identified as $\mathrm{CD}_{11 \mathrm{~b}^{+}}(\mathrm{P} 4)$ were more abundant in the $\mathrm{Cb}^{-/-}$mice than in the controls and expressed enhanced mRNA levels of Cb2r and $I l 1 b$ (Figure 5B).

The neuroinflammatory phenotype observed in the $\mathrm{Cb} 1^{-/-}$mice was also sensitive to minocycline treatment, since CD11b expression (Figure 5C) and the morphological changes highlighted by IBA1 staining of microglia (Figure 5D) were normalized in the cerebellum after subchronic minocycline administration. Moreover, enhanced mRNA expression of $\mathrm{Cb} 2 r$ and inflammatory mediators, such as 

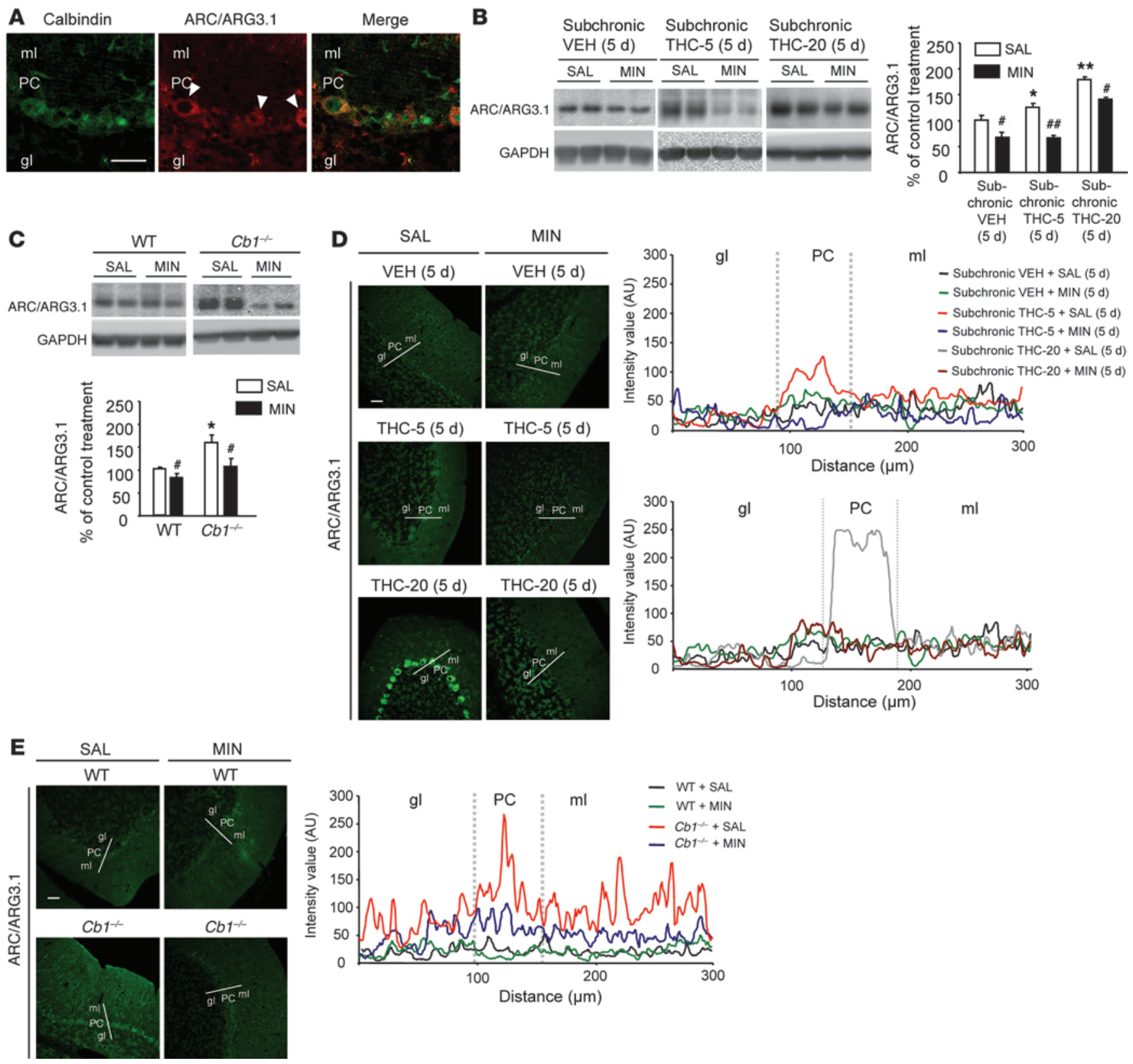

Figure 7

Cerebellar neuroinflammation secondary to cannabinoid downregulation modulates ARC/ARG3.1 expression. (A) Immunofluorescence detection of ARC/ARG3.1 and calbindin in Purkinje neurons in the cerebellar cortex. Scale bar: 50 um. (B) Immunoblot detection and quantification of ARC/ARG3.1 in cerebellar homogenates obtained at the end of subchronic exposure to MIN or SAL under subchronic THC (5 and $20 \mathrm{mg} / \mathrm{kg}$ ) and subchronic VEH treatment conditions ( $n=5-6$ mice per group). ${ }^{*} P<0.05$, ${ }^{* *} P<0.01$ versus subchronic VEH plus SAL ( 5 days); $P<0.05$, \#\# $P<0.01$ versus subchronic THC-5 plus SAL (5 days) or subchronic THC-20 plus SAL (5 days). (C) Immunoblot detection and quantification of ARC/ARG3.1 in cerebellar homogenates from $\mathrm{Cb}^{-1-}$ and WT mice at the end of subchronic exposure to MIN or SAL ( $n=5-6$ mice per group). ${ }^{*} P<0.05$ versus subchronic WT plus SAL; $P<0.05$ versus WT plus SAL. (D) ARC/ARG3.1 immunostained images from mice that were subchronically treated with THC-5, THC-20, or VEH and that received MIN or SAL for 5 days. ARC/ARG3.1 intensity was measured along a 300- $\mu \mathrm{m}$ line stretched along the 3 cerebellar layers. Plot represents ARC/ARG3.1 intensity alongside the layers quantified with ImageJ software. Scale bar: 100 um. (E) ARC/ARG3.1 immunostained images from $\mathrm{Cb}^{-1-}$ and WT mice at the end of subchronic exposure to MIN or SAL. ARC/ARG3.1 intensity was measured along a 300- $\mu \mathrm{m}$ line stretched along the 3 cerebellar layers. Plot represents ARC/ARG3.1 intensity alongside the layers quantified with ImageJ software. Scale bar: $100 \mu$ m.

Il1b, Il10, Tnfa, Cox2, and Cxcl2, was detected in the cerebellum of $\mathrm{Cb} 1^{-/-}$mice, and these changes were sensitive to minocycline treatment (Figure 5E). Additionally, no changes in GFAP expression were observed in the cerebellum of $\mathrm{Cb} 1^{-/-}$mice (Supplemental Figure 11).
The analysis of cerebellar function after minocycline treatment in $\mathrm{Cb}^{-/-}$mice revealed a remarkable recovery of the disrupted response in cerebellar conditioned learning (Figure 5F and Supplemental Figure 12A). Similarly, the motor coordination impair- 
A

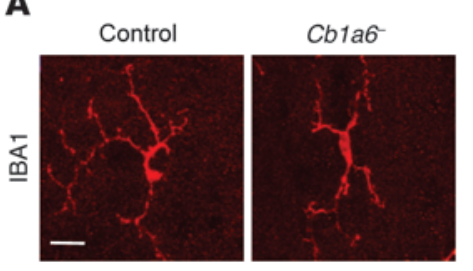

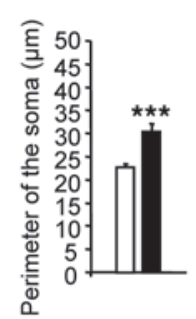

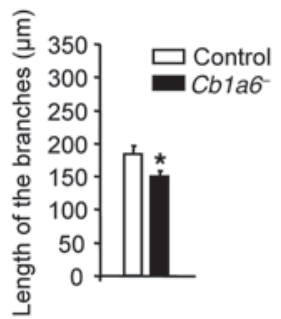

B

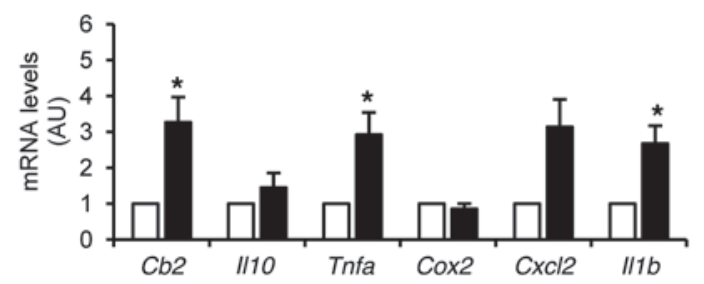

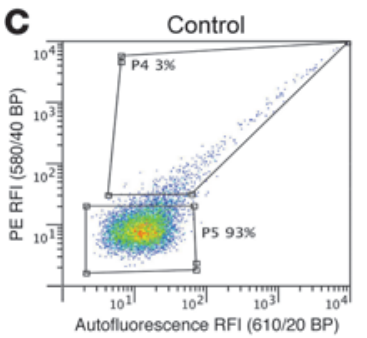
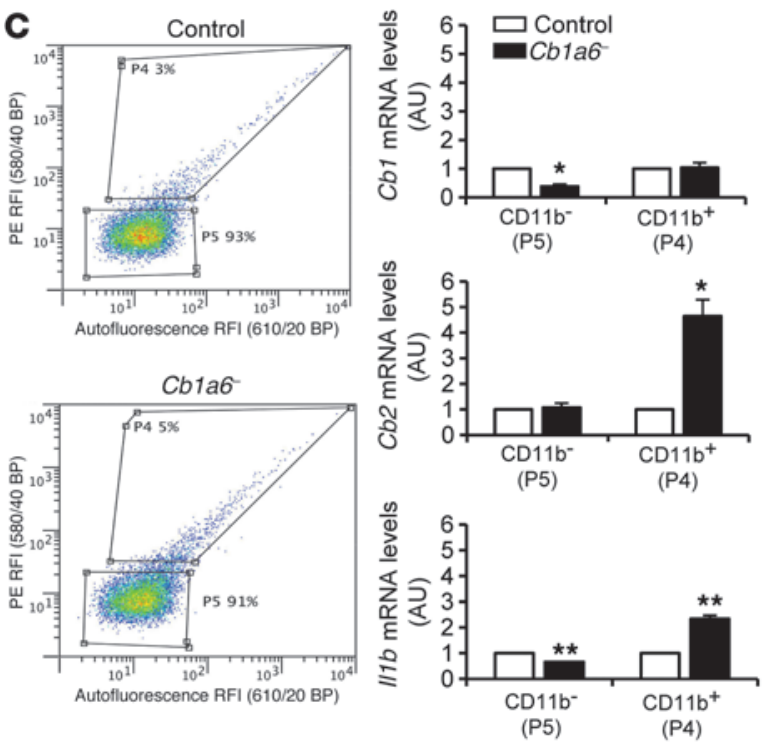
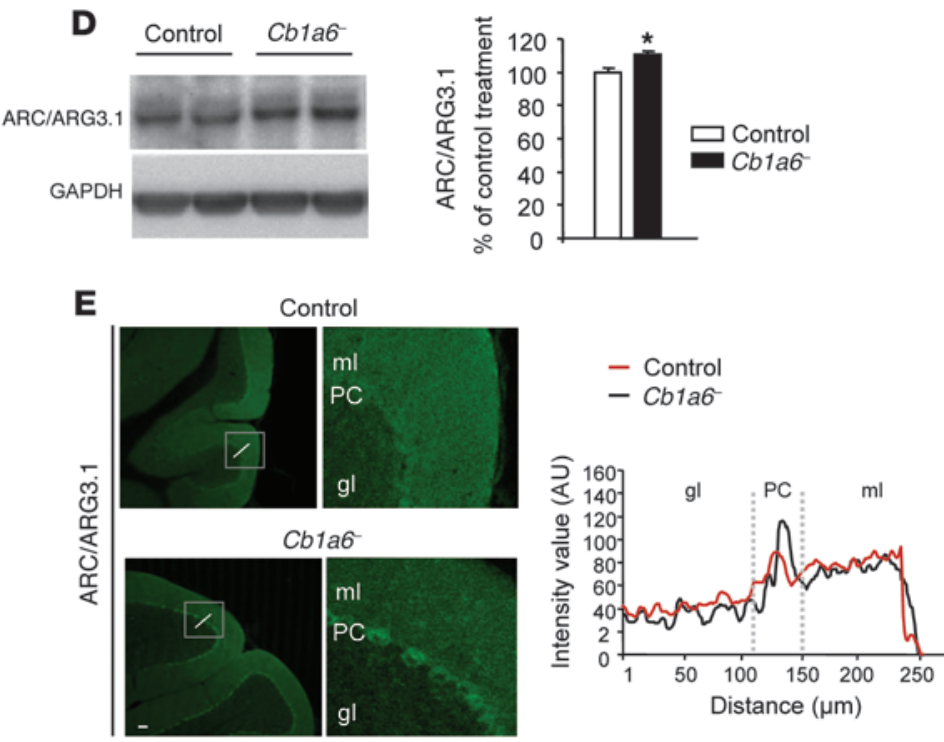

$\mathbf{F}$
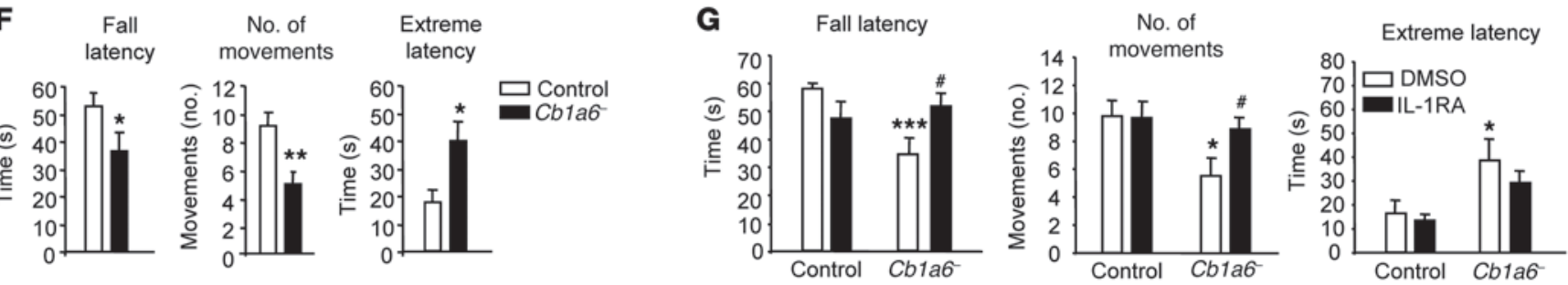

Figure 8

CB1R downregulation in the parallel fibers is sufficient to trigger cerebellar neuroinflammation and IL1-RA-sensitive motor coordination impairment. (A) Morphological analysis of IBA $1^{+}$cells in the cerebellar cortex of $C b 1 a 6^{-}$and control mice $(n=4-5$ per group, 6 cells per mouse). Scale bar: $25 \mu \mathrm{m}$. ${ }^{*} P<0.05,{ }^{* \star \star} P<0.001$ versus control. (B) Analysis of Cb2r mRNA expression by qRT-PCR and inflammation-related genes in the cerebellum of $\mathrm{Cb}_{\mathrm{ab}}{ }^{-}$and control mice ( $n=5$ per group). ${ }^{*} P<0.05$ versus control. (C) Flow cytometric analysis of CD $11 \mathrm{~b}$ expression and qRTPCR analysis of $\mathrm{Cb} 1 r, \mathrm{Cb} 2 r$, and $I / 1 b$ of acutely dissociated cerebellar cells from $C b 1 a 6^{-}$and control mice $\left(n=3-4\right.$ per group). ${ }^{*} P<0.05$ versus control. (D) Immunoblot detection and quantification of ARC/ARG3.1 in cerebellar homogenates from $\mathrm{Cb}_{12} 6^{-}$and control mice $(n=5-6$ mice per group). ${ }^{\star} P<0.05$ versus control. (E) ARC/ARG3.1 immunostaining in $\mathrm{Cb} \mathrm{a} \mathrm{6}^{-}$and control mice. Plot represents ARC/ARG3.1 intensity along the cerebellar layers. Scale bar: $100 \mu \mathrm{m}$. (F) Motor coordination analysis of $C b 1 a 6^{-}$and control mice $(n=11-15$ per group). (G) Motor coordination analysis with the coat-hanger test in $\mathrm{Cb} 1 \mathrm{a} 6^{-}$and control mice $(n=8-10$ per group). Four hours before the test, the mice received an injection of IL-1RA (100 mg/kg, i.p.) or its VEH (DMSO). ${ }^{\star} P<0.05,{ }^{\star \star} P<0.01,{ }^{\star \star \star} P<0.001$ versus control. ${ }^{\#} P<0.05$ versus $C b 1$ a6 $6^{-}$plus DMSO.

ment revealed in $\mathrm{Cb1}^{-/-}$mice by the coat-hanger test was also significantly improved with minocycline treatment (Figure 5G), demonstrating the relevance of microglial activation in the phenotype promoted by CB1R deletion.

IL-1 receptor signaling blockade resolves cannabinoid-mediated cerebellar deficits. As described above, cerebellar Il1b expression was enhanced 5 days after subchronic THC treatment cessation in $\mathrm{CD}_{11 \mathrm{~b}^{+}}(\mathrm{P} 4)$ cells, and was constantly altered in $\mathrm{Cb} 1^{-/-}$mice. Local microinjection of IL- $1 \beta$ into the cerebellum is known to produce ataxia (37) and to enhance the firing rates of cerebellar Purkinje neurons (38) acting on the IL-1 receptors (IL-1Rs) that are strongly expressed in Purkinje neurons (39). We therefore tested whether an inhibitor of IL-1R signaling, an end-protected L-valyl derivative with antagonistic effects on IL-1R (IL-1RA), would improve cerebellar performance under our experimental conditions. For delayed eyeblink conditioning analysis, mice received IL-1RA (100 $\mathrm{mg} / \mathrm{kg}$, i.p.) 4 days after subchronic THC treatment cessation for 3 consecutive days 4 hours before the test sessions (C5-C7) (Figure 
6A and Supplemental Figure 1C). Mice receiving IL-1RA showed an increase in the number of conditioned responses that reached statistical significance from the second day of IL-1RA administration for the THC $(5 \mathrm{mg} / \mathrm{kg})$ group and from the first day of administration for the THC (20 mg/kg) group (Figure 6A). Similarly, $\mathrm{Cb} 1^{-/-}$mice showed transiently improved performance in the delayed eyeblink conditioning paradigm after IL-1RA administration (Figure 6B and Supplemental Figure 12B). Correspondingly, acute IL-1RA administration 4 hours before the coat-hanger test normalized the performance of the mice that received subchronic THC ( 5 or $20 \mathrm{mg} / \mathrm{kg}$ ) treatment to that of the control mice (Figure 6C). A similar treatment with IL-1RA in $\mathrm{Cb} 1^{-/-}$mice significantly improved their performance in the coat-hanger test (Figure $6 \mathrm{D})$. Interestingly, no effect of IL-1RA was observed in the control groups (subchronic vehicle [5 days] and WT mice), either with the conditioned cerebellar learning test or the coat-hanger test (Figure 6). Moreover, no alteration of cerebellar microglia morphology was detected after acute IL-1RA administration (Supplemental Figure 13), pointing to a specific role of this blockade strategy under neuroinflammatory conditions.

Cerebellar neuroinflammation modulates ARC/ARG3.1 expression. The activity of cerebellar Purkinje neurons can be evaluated by measuring the expression of the activity-regulated cytoskeleton-associated protein, also known as ARG3.1 (ARC/ARG3.1) (40). ARC/ ARG3.1 is expressed in Purkinje cell neurons, where it colocalizes with calbindin immunoreactivity (Figure 7A). We measured ARC/ARG3.1 expression in cerebellar homogenates to assess the Purkinje cell activity under the experimental conditions studied above. Mice after subchronic THC treatment cessation (Figure 7B) and $\mathrm{Cb}^{1^{-/}}$mice (Figure 7C) showed enhanced expression of ARC/ ARG3.1 compared with control mice. Interestingly, a clear reduction of ARC/ARG3.1 expression was observed after subchronic minocycline treatment in both experimental conditions (Figure 7, B and C).

Immunofluorescence analysis for ARC/ARG3.1 revealed a localized increase in ARC/ARG3.1 expression in the Purkinje cells of the cerebellum both after subchronic THC treatment cessation (Figure 7D) and in $\mathrm{Cb}^{-/-}$mice (Figure 7E). This result was corroborated by the intensity analysis performed across the different layers of the cerebellar cortex (Figure 7, D and E). These results point to a relevant involvement of the neuroinflammatory process, which is produced by cerebellar CB1R downregulation, in the activity-related changes (expression of ARC/ARG3.1) promoted in Purkinje cells.

$C B 1 R$ deregulation in parallel fibers recreates the cerebellar deficit. To better evaluate whether the downregulation of CB1R in cerebellar parallel fibers would affect microglial responses and cerebellar performance, we used a conditional $\mathrm{Cb} 1 \mathrm{a} 6^{-}$mouse model lacking CB1R in cerebellar granular cells (12). In this mouse line, CB1Rs were absent from the parallel fibers, but were preserved in other cerebellar locations where CB1Rs are less abundantly expressed, such as the climbing fibers (Supplemental Figure 14). In this mouse line, immunofluorescence detection of CB1Rs on microglial cells located in the molecular layer of the cerebellum was negligible and indistinguishable from the background staining (Supplemental Figure 15). Microglial morphology revealed by staining with IBA1 showed an activated phenotype (Figure $8 \mathrm{~A}$ ) of these cells in the cerebellar molecular layer of the $\mathrm{Cb} 1 \mathrm{a} 6^{-}$mice. These mice displayed an enhanced cerebellar mRNA expression of Cb2r, Il1 b, and Tnfa (Figure 8B). The increased $\mathrm{Cb} 2 r$ and $I l 1 b$ expression was restricted to $\mathrm{CD}_{11 \mathrm{~b}^{+}}(\mathrm{P} 4)$ cells, but $\mathrm{Cb} 2 \mathrm{r}$ and $\mathrm{Il} 1 \mathrm{~b}$ were not detected in CD11b(P5) cells acutely isolated from the cerebellum (Figure 8C). Similar to previous data involving $\mathrm{Cb} 1^{-/-}$mice, $\mathrm{Cb} 1 \mathrm{a} 6^{-}$mice showed enhanced expression of cerebellar ARC/ARG3.1 (Figure 8, D and E) and, compared with their control littermates, exhibited a significant motor coordination deficit in the coat-hanger test (Figure $8 \mathrm{~F}$ ) that was prevented by acute IL-1RA administration (Figure 8G). The use of this novel genetic tool provides direct evidence for the role of CB1R in cerebellar parallel fibers in modulating these morphological and behavioral responses.

\section{Discussion}

Our study describes the local activation of cerebellar microglia as a result of dysregulated CB1R activity produced by both subchronic THC exposure and CB1R genetic disruption. This microglial activation correlates with functional deficits in cerebellar conditioned learning and motor coordination. The microglial activation was typified by alterations in microglial morphology, mainly in the molecular layer of the cerebellum, and was accompanied by the enhanced expression of specific proinflammatory genes, such as Il1b. Pharmacological blockade of microglial activation and IL-1R signaling prevented the deficit in cerebellar associative learning and motor coordination in subchronically THC-treated mice and in $\mathrm{Cb}^{-/-}$mice. Altogether, these results reveal the critical role of microglia-mediated signaling in the cerebellar dysfunctions associated with CB1R deregulation.

Microglial cells play an important role in innate immune responses in the CNS $(21,24)$. Under pathological conditions, microglial cells are activated through complex processes that produce remarkable changes in their morphology and gene expression (26, 29, 30). Cessation of subchronic THC treatment and genetic disruption of CB1R selectively produced in the cerebellum an increase in the expression of the microglial activation marker CD11b, an enhanced expression of proinflammatory cytokines, and a microglial morphological alteration occurring mainly in the cerebellar molecular layer. Sorted $\mathrm{CD}_{11 \mathrm{~b}^{+}}(\mathrm{P} 4)$ cerebellar cells from these mice showed an increased expression of $C b 2 r$ and $I l 1 b$ mRNA, an effect not observed in CD11 $\mathrm{b}^{-}$ (P5) cells. Under these experimental conditions, CB1R expression was enhanced specifically on the CD11b- (P5) cells, but not on the $\mathrm{CD}_{11} \mathrm{~b}^{+}(\mathrm{P} 4)$ cells. This result correlates with that obtained for $\mathrm{Cb} 1 \mathrm{r}$ mRNA expression under similar THC treatment conditions in rat cerebellum (41). This microglial activation was not associated with cellular stress, as revealed by the Fluoro-Jade B assay. Microglial cells in resting conditions present low to undetectable levels of CB1R and CB2R mRNA and protein (20). Therefore, the microglial activation process triggered by THC is unlikely to be mediated by the direct effect of THC on the brain microglial population, and it is likely related to the deregulation of CB1R function specifically in the cerebellum. In contrast to CB1R protein downregulation, cerebellar Cb2r mRNA was upregulated in parallel with the development of the neuroinflammatory phenotype in $\mathrm{CD}_{11} \mathrm{~b}^{+}$cells. It is plausible that THC, acting as an agonist on microglial CB2Rs, may reduce microglial activation through CB2R modulation $(42,43)$ during exposure to THC. After THC cessation, microglia would be released from the CB2R-mediated inhibition of this cannabinoid agonist. Under these conditions, microglia would remain activated while CB1R functionality is recovered. In agreement with the selectivity of this response, Cb1r genetic removal was sufficient to induce a similar change in the microglial phenotype in the cerebellum, but not in the hippocampus. In this regard, a previous study reported that 12 -month-old $\mathrm{Cb}^{-1-}$ 
mice, but not 2- or 5-month-old $C b 1^{-/-}$mice, displayed enhanced neuroinflammation in the hippocampus (44). The microglial activation reported here in the cerebellum of 2- to 3-month-old constitutive $\mathrm{Cb}^{-/-}$and $\mathrm{Cb} 1 \mathrm{a} 6^{-}$mice reveals the particular sensitivity of this brain area to the neuroinflammatory process promoted by the downregulation of CB1R activity.

We evaluated the relationship between microglial activation and cerebellar deficits using minocycline, a second-generation tetracycline antibiotic with inhibitory effects on microglial activation (35) that are probably due to its ability to block 5-lipoxigenase expression (45). Minocycline patently reduced cerebellar conditioned learning and motor coordination deficits in the THC-withdrawn and $\mathrm{Cb}^{-/-}$mice, together with the landmarks of microglial activation. Notably, this improvement in cerebellar function was not associated with a recovery of CB1R density in the cerebellum of mice after subchronic THC treatment cessation. Moreover, minocycline was effective at improving cerebellar conditioned learning and motor coordination in mice lacking CB1Rs, pointing to a sofar-unknown role for microglial reactivity status in cerebellar performance.

Microglial activation was associated with enhanced expression of $\mathrm{Cb} 2 r$ and various proinflammatory factors, such as $I l 1 b$, in the cerebellum of mice subchronically treated with THC and in $\mathrm{Cb} 1^{-/-}$ mice. This result was supported by the enhanced expression of $\mathrm{Cb} 2 r$ and $\mathrm{Il} 1 \mathrm{~b}$ in $\mathrm{CD} 11 \mathrm{~b}^{+}$cells, but not in $\mathrm{CD}^{11 \mathrm{~b}^{-}}$cells sorted after cerebellar cell dissociation in THC-exposed mice and in mice with genetic disruptions of CB1R expression. IL- $1 \beta$ is mainly produced in the brain by microglia (46), and it has been reported to directly regulate Purkinje cell activity (38). IL-1RA administration induced a recovery of cerebellar conditioned learning and motor coordination in THC-withdrawn and $\mathrm{Cb}^{-/-}$mice. In this mouse line, both minocycline and IL-1RA substantially improved cerebellar conditioned learning and motor coordination performance, a result that reveals the crucial involvement of microglial activation in cerebellar functionality. Thus, the neuroinflammation produced as a consequence of CB1R downregulation would be a key factor in the cerebellar functional deficits reported previously $(17,36)$ and herein.

The cerebellar conditioned responses recorded in the eyeblink conditioning test for THC-withdrawn mice and $\mathrm{Cb}^{-/-}$mice that received IL-1RA treatment were markedly different on the last conditioning days. Indeed, IL-1RA treatment produced a transitory improvement of conditioned responses in $\mathrm{Cb}^{-/-}$mice only during treatment, whereas conditioned response improvement in THC-withdrawn mice was observed even beyond the IL-1RA treatment. This result revealed the recovery of CB1R functionality in THC-withdrawn mice at the end of the conditioning period compared with the $\mathrm{Cb1^{-/- }}$ mice, in which the CB1Rs were permanently eliminated. IL-1RA administration had immediate effects on the performance of cerebellar conditioned learning, and these effects were more relevant in those conditions in which there was markedly strong microglial activation, such as in the high-THC-dose $(20 \mathrm{mg} / \mathrm{kg})$ mice or the $\mathrm{Cb} 1^{-/-}$mice. In contrast, the effects of minocycline did not appear after a single administration and were only evident after the second or third day of treatment. This is in agreement with the idea that IL- $1 \beta$ acts as the effector that is produced by activated microglia and is involved in the alteration of cerebellar function and that minocycline may instead be associated with a switch in microglial activation status to a nonreactive state in which IL- $1 \beta$ expression is reduced. Notably, the direct action of IL-1 $\beta$ on Purkinje cells has been associated with an increase in cellular excitability that could directly affect cerebel- lar output (38). The enhanced activity of Purkinje neurons under neuroinflammatory conditions fits with the increased expression of cerebellar ARC/ARG3.1 protein reported here and proposed previously (40). The increase in Purkinje neuronal activity may affect the functionality of their projecting area, leading to deficits in specific cerebellar functions.

A possible mechanism to explain this neuroinflammatory process would be the putative glutamate mishandling by CB1R-containing deregulated terminals, since extracellular glutamate concentrations are tightly regulated at synapses onto Purkinje cells (47). Indeed, the excitatory transmission to Purkinje cells shows an extended presence of glutamate in the synaptic cleft (48). Moreover, the endocannabinoid system is strongly activated in the case of glutamate spillover at this particular synapse in order to rapidly shut down glutamate release (49). In agreement with these observations, increased postsynaptic excitatory activity has been reported in cerebellar Purkinje cells after subchronic THC-mediated CB1R downregulation (14). In addition, different forms of synaptic plasticity between parallel fibers and Purkinje neurons mediated by glutamate are suppressed in $\mathrm{Cb}^{-/-}(11)$ and $\mathrm{Cb} 1 \mathrm{a} 6^{-}$mice (12).

Our results suggest that the neuronal/glial circuitry in the cerebellum is particularly sensitive to conditions affecting CB1R functionality. In this regard, it was shown that chronic cannabis consumers assessed during periods of abstinence demonstrate hypoactive cerebellar activity (50), which could be interpreted as a consequence of CB1R downregulation in the cerebellar cortex. Indeed, chronic cannabis consumption in humans produced a clear downregulation in brain CB1R mRNA levels as determined via postmortem $\left[{ }^{3} \mathrm{H}\right]$-rimonabant binding (9). These results uncover a new neurobiological mechanism for the deleterious effects of THC on cerebellar function that may underlie the recent observations of cerebellar motor learning acquisition in current (15) and former (18) cannabis users. The neuronal circuits involved in the eyeblink conditioning response are the same in mice and humans, thus demonstrating the translational relevance of this test.

Therefore, we hypothesize (Supplemental Figure 16) that these cerebellar neuroinflammatory alterations induced as a consequence of CB1R downregulation represent the neurobiological basis for this side effect related to cannabis consumption. Interestingly, a more difficult-to-acquire learning paradigm, trace eyeblink conditioning, which requires forebrain function, was not affected in cannabis users (19), pointing to the specific disruption promoted by repeated THC exposure in this form of cerebellar learning. The reversal of these cerebellar deficits in our animal models by blocking microglial activation and IL-1RA offers interesting new therapeutic approaches for treating these cannabis-induced side effects.

\section{Methods}

\section{Animals}

Seven to 10 -week-old male mice were used. WT Swiss albino mice (CD-1) were purchased from Charles River Laboratory. $\mathrm{Cb} 1^{-/-}$mice on a CD-1 background (51) and $\mathrm{Cb}_{1 \mathrm{a} 6^{-}}$mice on a C57BL/6 background (12) were bred at our animal facility. Both littermate- and age-matched controls were used and found to be indistinguishable; data for controls were thus pooled. Gabra6Cre and Cb1/f/f animals were maintained on a C57BL/6-J background as described previously (52). Gabra6cre; $\mathrm{Cb} 1^{\mathrm{f} / \mathrm{f}}$ males were mated with $\mathrm{Cb} 1 \mathrm{f} / \mathrm{f}$ females to generate Gabra6cre; $\mathrm{Cb} 1 \mathrm{f} / \mathrm{f}$ experimental animals and $\mathrm{Cb} 1 \mathrm{fff}$ littermate controls. After arrival to the facility, mice were 
housed in plastic cages with 4 mice per cage and maintained in a controlled temperature $\left(21^{\circ} \mathrm{C} \pm 1^{\circ} \mathrm{C}\right)$ and humidity $(55 \pm 10 \%)$ environment. Food and water were available ad libitum. Lighting was maintained at 12 -hour cycles (on at 8 am and off at $8 \mathrm{pm}$ ). All the experiments were performed during the light phase of the light/dark cycle. The animals were habituated to the experimental room and handled for 1 week before the start of the experiments. All behavioral experiments were conducted by an observer blind to the experimental conditions.

\section{Drugs and treatments}

THC was purchased from THC Pharm GmbH; cremophor-EL and minocycline (MIN) were purchased from Sigma-Aldrich; rimonabant (RIM) was provided by Sanofi-Aventis Recherche, and IL-1R antagonist (IL-1RA) was purchased from Merck Millipore. THC and RIM were diluted in vehicle (VEH) solution (5\% ethanol, 5\% cremophor-EL, $90 \%$ saline [SAL]). MIN was dissolved in $0.9 \%$ SAL. IL-1RA was dissolved in DMSO. THC, RIM, MIN, and their vehicles were administered i.p. in a volume of $0.1 \mathrm{ml} / 10 \mathrm{~g}$ of body weight. IL-1RA and its vehicle (DMSO) were administered i.p. at a dose of $0.02 \mathrm{ml} / 10 \mathrm{~g}$ of body weight. Subchronic THC (or VEH as a control) was administered according to a protocol that produced cannabinoid physical dependence (31). Thus, the mice were injected with THC $(1 \mathrm{mg} / \mathrm{kg}, 2.5 \mathrm{mg} / \mathrm{kg}, 5 \mathrm{mg} / \mathrm{kg}$, or 20 $\mathrm{mg} / \mathrm{kg}$, i.p.) or VEH twice daily at 09:00 and 19:00 hours for 5 consecutive days. On the sixth day, the mice received only the morning injection of THC or VEH. The mice were sacrificed 4 hours after this last injection (subchronic THC-5, subchronic THC-20, and subchronic VEH groups). To study the effects of spontaneous cannabinoid withdrawal, the mice were analyzed 5 days after the end of the cannabinoid or VEH treatment: subchronic THC-1 (5 days), subchronic THC-2.5 (5 days), subchronic THC-5 (5 days), subchronic THC-20 (5 days), and subchronic VEH (5 days) groups.

To evaluate the precipitated THC withdrawal, mice received RIM $(10 \mathrm{mg} / \mathrm{kg}$, i.p.) 4 hours after the last dose of THC (subchronic THC plus RIM [ 5 days] group) or VEH (subchronic VEH plus VEH [ 5 days] group). Mice were sacrificed 5 days later.

To assess the role of microglial activation in cerebellar function after the spontaneous withdrawal of subchronic THC exposure, mice were first treated subchronically with THC $(5 \mathrm{mg} / \mathrm{kg}$ or $20 \mathrm{mg} / \mathrm{kg})$ or VEH. On the sixth day, and 3 hours after the last VEH or THC injection, mice received the first administration of MIN ( $40 \mathrm{mg} / \mathrm{kg}$, i.p., once per day for 5 days). The experimental groups were: subchronic VEH plus MIN (5 days) and subchronic VEH plus SAL (5 days); subchronic THC-5 plus MIN (5 days) and subchronic THC-5 plus SAL (5 days); subchronic THC-20 plus MIN (5 days); and subchronic THC-20 plus SAL (5 days). Similarly, Cb1 $1^{-/-}$mice and WT controls were treated for 5 days with MIN (40 mg/kg, i.p., once per day). The experimental groups were: WT plus SAL and WT plus MIN, and $\mathrm{Cb}^{-/-}$plus SAL and $\mathrm{Cb}^{-/-}$plus MIN. The mice were analyzed for conditioned cerebellar learning, as described in Supplemental Figure 1B and Supplemental Figure 12A, and for motor coordination at the end of subchronic MIN treatment. Afterward, mice were sacrificed for biochemical or immunohistochemical assays.

To study the effect of IL-1RA ( $100 \mathrm{mg} / \mathrm{kg}$, i.p.) on conditioned cerebellar learning, mice were treated subchronically with VEH, THC-5, and THC-20 (for 5 days, twice per day, and once on the sixth day). Four days after the last VEH, THC-5, or THC-20 injection, they received an acute administration of IL-1RA or DMSO (see Supplemental Figure 1C). In addition, $\mathrm{Cb} 1^{-/-}$mice and WT controls were studied for the effects of IL-1RA on cerebellar conditioned learning (see Supplemental Figure 12B). Four hours after IL-1RA administration, the mice were assayed using the eyeblink conditioning paradigm, and this procedure was repeated on 3 consecutive days (sessions C5-C7). In another set of animals, IL-1RA or DMSO was injected acutely into THC-withdrawn mice, $\mathrm{Cb} 1^{-/-}, \mathrm{Cb} 1 \mathrm{ab}^{-}$, and control mice to study motor coordination using the coat-hanger test. In this case, mice were subsequently sacrificed for biochemical and immunohistochemical analysis.

\section{Immunoblot analysis}

Frozen cerebellar, striatal, cortical, or hippocampal tissues were processed as previously reported (53). For immunoblotting, we used the following antibodies: anti-CD11b (rabbit, 1:500) from Abcam; antiARC/ARG3.1 (mouse, 1:100) and anti-GAPDH (mouse, 1:5,000) from Santa Cruz Biotechnology; anti-CB1R (rabbit, 1:1,000) from Frontier Science; anti-IBA1 (rabbit, 1:500) from Wako Pure Chemical Industries; and anti-glial fibrillary acidic protein (GFAP) (rabbit, 1:500) from Dako. Blots containing equal amounts of cerebellar protein samples $(40 \mu \mathrm{g} /$ lane) were probed with different primary antibodies. Bound primary antibodies were detected with HRP-conjugated antibodies against mouse (diluted at 1:5,000; Thermo Fisher Scientific) and against rabbit (diluted at 1:10,000; Cell Signaling Technologies). Both antibodies were visualized by enhanced chemiluminescence detection (SuperSignal West Femto; Thermo Fisher Scientific). When necessary, Immobilon-P membranes (Millipore) were stripped before reblocking and reprobing. The optical density of the relevant immunoreactive bands was quantified after acquisition on a ChemiDoc XRS system (Bio-Rad) controlled by Quantity One software v4.6.3 (Bio-Rad). The quantification of protein expression was in the linear range for the experimental conditions used in this study (data not shown). For quantitative purposes, the optical density values for the proteins of interest were normalized to the detection of the housekeeping control GAPDH in the same samples and expressed as a percentage of the control treatment.

\section{Tissue preparation for immunohistochemistry and immunofluorescence}

After pharmacological treatment and/or behavioral testing, the mice were deeply anesthetized by i.p. injection $(0.2 \mathrm{ml} / 10 \mathrm{~g}$ of body weight) of a mixture of ketamine $(100 \mathrm{mg} / \mathrm{kg})$ and xylazine $(20 \mathrm{mg} / \mathrm{kg})$ prior to intracardiac perfusion of $4 \% \mathrm{PFA}$ in $0.1 \mathrm{M} \mathrm{Na}_{2} \mathrm{HPO}_{4} / \mathrm{NaH}_{2} \mathrm{PO}_{4}$ buffer, $\mathrm{pH} 7.5$, delivered with a peristaltic pump at 19 milliliters per minute for 5 minutes. Brains were removed and postfixed overnight at $4{ }^{\circ} \mathrm{C}$ in the same fixative solution. Brain sections $(30-\mu \mathrm{m})$ were obtained with a vibratome (Leica) and kept in a solution containing 30\% ethylene glycol, 30\% glycerol, and $0.1 \mathrm{M} \mathrm{PB}$ at $-20^{\circ} \mathrm{C}$ until processed for immunohistochemical or immunofluorescence analysis.

\section{Immunohistochemistry}

The immunohistochemical procedure was performed as previously described (54). The antibody against GFAP was used at a 1:500 dilution in this procedure.

\section{Immunofluorescence}

Free-floating slices were rinsed in TBS (0.25 M Tris, $0.15 \mathrm{M} \mathrm{NaCl}, \mathrm{pH} 7.5)$, incubated for 15 minutes in $0.2 \%$ Triton X-100 in TBS, and then incubated overnight at $4{ }^{\circ} \mathrm{C}$ with the following primary antibodies: anti-IBA1 (1:500), anti-CD11b (1:50), both from Cederlane Laboratories Limited; and anti-CB1R (1:1,000), anti-calbindin (1:300), and anti-ARC/ARG3.1 (1:100) from Santa Cruz Biotechnology. Double staining was performed with anti-CB1R (rabbit, 1:1,000) from Frontier Science and anti-VGLUT1 (mouse, 1:500) from Merck Millipore. The next day (16 hours later), after 2 rinses in TBS, sections were incubated for 2 hours at room temperature with fluorescent anti-rabbit-Cy3 (1:500; Jackson ImmunoResearch Lab- 
oratories) or biotinylated anti-mouse antibody (1:400; Vector Laboratories). After a 10-minute wash, the slices were incubated for 15 minutes with streptavidina-Alexa Fluor 488 (Invitrogen). After two 10-minute washes, tissue sections were mounted onto gelatin-coated slides with Mowiol mounting media (0.5 M Mowiol 40-88 [Sigma-Aldrich], 20\% glycerol, 0.1 M Tris $\mathrm{pH} 8.5$ ).

Confocal images were obtained using a Leica SP2 confocal microscope, adapted to an inverted Leica DM IRBE microscope. Alexa Fluor 488 (Invitrogen) and Cy3 were excited with the 488-nm line of an argon laser and the 543-nm line of a green neon laser, respectively. For double immunofluorescence, images were taken of each animal in a sequential mode. All further image analysis was performed by an experienced observer blind to the experimental conditions.

$C D 11 b^{+} I B A 1^{+}$microglia quantification. Stained sections were analyzed by confocal microscopy. We used the specific "particle analysis, cell counter" tool from ImageJ software (NIH) for the manual quantification of CD11 $\mathrm{b}^{+}$ IBA $1^{+}$positive microglial cells. Twenty to 25 images from 4 to 5 animals (5 images per animal) were analyzed for each experimental group. We used this method to determine the number of $\mathrm{IBA} 1^{+}$and IBA $1^{+} \mathrm{CD} 11 \mathrm{~b}^{+}$microglial cells after the spontaneous and precipitated THC withdrawal.

CD11b/IBA1 colocalization analysis. Confocal images were taken for each animal $(n=5)$ in a sequential mode. We determined a constant region of interest (ROI) surrounding the microglial cells $(n=3$ mice per group; 5 images per mouse) that was large enough to encompass all of the cell ramifications with the cell body in the center (similar to that shown in Supplemental Figure 17). Afterward, the intensity correlation quotient (ICQ) between the CD11b and IBA1 signal within each ROI was calculated with ImageJ software (NIH).

CB1R intensity analysis. Confocal images (4 images per animal corresponding to the molecular and granular layers of the cerebellum) were acquired ( $n=4-5$ mice; $4-5$ images per mouse) in a sequential mode. Afterward, the CB1R signal intensity was evaluated using a constant ROI with Image software (NIH).

Morphological analysis. To evaluate the changes in microglial morphology, confocal microscopic images of whole microglial cells stained with IBA1 were acquired with an oil immersion lens ( $\times 40$ objective; 1.5 zoom). Images were taken at different $z$ levels $(0.8-\mu \mathrm{m}$ depth intervals) to evaluate the morphology of the whole cell. Afterward, the length of the microglial ramifications and the perimeter of the microglial soma were analyzed with ImageJ software (NIH) (see Supplemental Figure 17 for details). In all cases, the number of microglial cells analyzed was $16-24$ per group $(n=4-5$ mice; 4 cells per animal).

ARC/ARG3.1 intensity representation. To evaluate ARC/ARG3.1 expression in the cerebellum, confocal images were analyzed using ImageJ software (Plot Profile tool; NIH) for ARC/ARG3.1 signal intensity (gray value) along a $300-\mu \mathrm{m}$ line. For this purpose, a $300-\mu \mathrm{m}$ line was drawn through the 3 cerebellar layers: granular layer (gl), Purkinje cell (PC) layer, and molecular layer $(\mathrm{ml})$, and the intensity of each pixel on that line was represented.

\section{Fluoro-Jade B assay}

Cellular stress was evaluated with the Fluoro-Jade B assay (Chemicon) following the manufacturer's instructions. To determine whether cell death was accompanied by a microglial activation process, IBA1 immunofluorescence was performed, followed by the Fluoro-Jade B assay.

\section{Dissociation of cerebellar cells and CD11b-based cell sorting}

Cerebellar tissue from vehicle (5 days), THC-5 (5 days), and THC-20 (5 days) groups, as well as from $\mathrm{Cb}^{-/}{ }^{-}$, WT, $\mathrm{Cb} 1 \mathrm{a} 6^{-}$, and control mice $(n=3-4$ per group) was removed after anesthesia and was subjected to a 3 -minute perfusion with ice-cold $0.1 \mathrm{M} \mathrm{PB}, 0.9 \% \mathrm{NaCl}$. Tissues were enzymatically digested with a Neuronal Tissue Dissociation kit (Papain; Miltenyi Biotec) according to the manufacturer's instructions. The cell suspension in HBSS was stained with PE-conjugated anti-CD11b (Miltenyi Biotec). Phycoerythrin (PE) relative fluorescence intensity (PE RFI) was detected through a 580/40-nm band pass (BP) filter, and autofluorescence was detected through a $610 / 20-\mathrm{nm}$ BP filter. Cell sorting was performed to isolate the $\mathrm{CD}_{11 \mathrm{~b}^{+}}(\mathrm{P} 4)$ and CD11b- (P5) population. CD11b ${ }^{+}$(P4) cells were mainly positive for IBA1 staining (Supplemental Figure 8 ). Both the $\mathrm{CD}_{11 \mathrm{~b}^{+}(\mathrm{P} 4)}$ and CD11b- (P5) fractions were collected and used for further analyses of gene expression by RT-PCR.

\section{RNA extraction and reverse transcription}

Cerebellar tissues and CD11b+ (P4) and CD11b- (P5) sorted cells were collected and stored at $-80^{\circ} \mathrm{C}$. Isolation of total RNA was performed using an RNeasy Mini kit (tissue; QIAGEN) or the RNeasy Micro kit (dissociated cells; QIAGEN) according to the manufacturer's instructions. The quality of the total RNA was assessed by the spectrophotometric ratio of A260/ A280 (1.9:2.1). Total RNA concentration was measured using a NanoDrop spectrophotometer (Thermo Fisher Scientific).

Reverse transcription was performed with $0.3 \mu \mathrm{g}$ of total RNA from each animal to produce cDNA in a $10-\mu \mathrm{l}$ reaction with 200 units of SuperScript III Reverse Transcriptase (Invitrogen) and 500-ng oligo(dT) 15 primers. Reverse transcriptase reactions were carried out at $25^{\circ} \mathrm{C}$ for 10 minutes, then 50 minutes at $42^{\circ} \mathrm{C}$, and 15 minutes at $70^{\circ} \mathrm{C}$. The cDNAs from cerebellar tissues were diluted $1: 4$ and stored at $-20^{\circ} \mathrm{C}$ until use, while cDNAs from $\mathrm{CD} 11 \mathrm{~b}^{+}$(P4) and CD11b- (P5) cells were stored without further dilution until use.

\section{Quantitative real-time $P C R$ analysis}

Real-time PCR was carried out with an ABI PRISM 7700 Sequence Detection System (Applied Biosystems) using the SYBR Green PCR Master Mix (Applied Biosystems) according to the manufacturer's protocol. All the samples were tested in triplicate, and the relative expression values were normalized to the expression value of GAPDH.

The following primers specific for mouse Cb2r (sense, $5^{\prime}$-GGTCGACTCCAACGCTATCTTC-3'; antisense, 5'-GTAGCGGTCAACAGCGGTTAG-3'), Il1b (sense, 5'-GAAGAGCCCATCCTCTGTGACT-3'; antisense, 5'-GTTGTTCATCTCGGAGCCTGTAG-3'), Tnfa (sense, 5'-GACTAGCCAGGAGGGAGAACAG-3'; antisense, 5'-CAGTGAGTGAAAGGGACAGAACCT-3'), Cox2 (sense, 5'-GGCCGACTAAATCAAGCAACA-3'; antisense, 5' -CAATGGGCATAAAGCTATGGTTAGA-3'), Cxcl2 (sense, 5'-ACTGCATCTGCCCTAAGGTCTT-3'; antisense, 5'-TGCTTGAGGTGGTTGTGGAA-3'), Cd11b (sense, 5'-GGATCCGGAAAGTAGTGAGAGAAC-3'; antisense, 5'-CCGAATTTTTCTCCATCTGTGAT-3'), Illo (sense, 5'-GGCGCTGTCATCGATTTCTC-3'; antisense, 5'-GCCTTGTAGACACCTTGGTCTTG-3'), and Gapdh (sense, 5'-ATGACTCCACTCACGGCAAAT-3'; antisense, 5'-GGGTCTCGCTCCTGGAAGAT-3') were used as endogenous housekeeping controls to standardize the amount of target cDNA. Amplified PCR products were separated on a $2 \%$ agarose gel and stained with ethidium bromide to confirm the specificity of the primers.

Samples were analyzed by the $\Delta \Delta \mathrm{Ct}$ method. $\Delta \Delta \mathrm{Ct}$ values were calculated as the $\Delta \mathrm{Ct}$ of each test sample (different pharmacological treatments) minus the mean $\Delta \mathrm{C}$ t of the calibrator samples (VEH-SAL group) for all the genes analyzed. The fold change was calculated using the equation $2^{(-\Delta \Delta C \mathrm{Ct})}$.

\section{Somatic expression of THC withdrawal}

The somatic signs of THC withdrawal were precipitated 4 hours after the last THC or vehicle injection (31). Briefly, mice were placed in a circular clear plastic observation area $(30 \mathrm{~mm}$ diameter $\times 35 \mathrm{~mm}$ height $)$ for a 
15-minute period of habituation. Immediately after habituation, the animals were observed for another 15 minutes, followed by administration of rimonabant $(10 \mathrm{mg} / \mathrm{kg}$, i.p.). Then, mice were observed for a 45 -minute period. Observations of somatic signs before and after rimonabant challenge were made at 5-minute intervals. A global withdrawal score was then inferred (31).

\section{Delayed eyeblink conditioning}

Mice assigned for classical conditioning of eyelid responses were prepared as follows: first, they were anesthetized with $0.8 \%$ to $1.5 \%$ isoflurane, supplied from a calibrated Fluotec 5 (Ohmeda) vaporizer, at a flow rate of 1 to 4 liters of oxygen per minute (AstraZeneca) and delivered by a mouse anesthesia mask (David Kopf Instruments). Once anesthetized, the animals were implanted with bipolar recording electrodes in the left orbicularis oculi muscle and with stimulating electrodes on the ipsilateral supraorbital nerve. Electrodes were made of $50-\mu \mathrm{m}$, Teflon-coated, annealed stainless steel wire (A-M Systems). The electrode tips were stripped of the isolating cover for $0.5 \mathrm{~mm}$ and bent as a hook to allow for stable insertion into the upper eyelid. A bare silver wire affixed to the skull served as a ground. All the implanted wires were soldered to two 4-pin sockets (RS Amidata). Bone screws and dental cement fixed the cannula and the sockets to the skull.

After implantation of the stimulating and recording electrodes, mice were randomly assigned to the experimental groups (see Supplemental Figure 1 and Supplemental Figure 12 for detailed chronogram). All experimental groups, WT, $\mathrm{Cb1}^{--}$, VEH, and THC-1/2.5/5/20, were familiarized with the experimental chambers for 30 minutes over 3 consecutive days. During this period, the intensity thresholds for activation of the supraorbital nerve and the proper implantation of recording electrodes in the orbicularis oculi muscle were checked. Then, mice were habituated to the tone (conditioned stimulus) for 2 consecutive days. Afterward, the conditioning sessions were performed once daily for 9 consecutive days starting the next day after the second habituation day $(\mathrm{H} 2)$. The first conditioning session was performed on the sixth day of THC treatment, coinciding with the first day of MIN (or SAL) treatment (Supplemental Figure 1B). In the case of $\mathrm{Cb}^{-1-}$ and WT mice, the conditioning sessions and the administration of SAL or MIN were conducted according to the schedule described in Supplemental Figure 12A. The conditioning sessions started 1 hour after MIN (or SAL) administration.

IL-1RA (100 mg/kg, i.p.) or its vehicle (DMSO), were administered on 3 consecutive days (C5, C6, C7), 4 days after the end of subchronic cannabinoid treatment (Supplemental Figure 1C and Supplemental Figure 12B). Conditioned sessions started 4 hours after IL-1RA or DMSO administration.

The percentage of conditioned responses was computed every day over a 9-day period. Experimental sessions were carried out with 4 animals at a time. Animals were placed in separate, small $(5 \times 5 \times 10 \mathrm{~cm})$ plastic chambers located inside a larger $(30 \times 30 \times 20 \mathrm{~cm})$ Faraday box. Electromyographic activity of the orbicularis oculi muscle was recorded with Grass P511 differential amplifiers (Grass-Telefactor), at a bandwidth of $0.1 \mathrm{~Hz}$ to $10 \mathrm{kHz}$.

Classical eyeblink conditioning was achieved with the use of a delayed conditioning paradigm. A tone $(370 \mathrm{~ms}, 600 \mathrm{~Hz}, 90 \mathrm{~dB})$ was used as a conditioned stimulus (CS) and was followed $270 \mathrm{~ms}$ from its onset by a $500-\mu \mathrm{s}$, $\times 3$ threshold, square, cathodal pulse applied to the supraorbital nerve as an unconditioned stimulus (US). Thus, the tone and the pulse terminated simultaneously. A conditioning session consisted of 60 CS-US presentations and lasted approximately 30 minutes. For a proper analysis of conditioned responses, the CS was presented alone in $10 \%$ of the cases. CS-US presentations were separated at random by $30 \pm 5$ seconds. For habituation sessions, only the CS was presented, also 60 times per session, at intervals of $30 \pm 5$ seconds. As a criterion, we considered a "conditioned response" the presence, during the CS-US interval, of electromyographic activity lasting more than $20 \mathrm{~ms}$ and initiated more than $50 \mathrm{~ms}$ after CS onset.

\section{Motor coordination tests}

Rod test. The equilibrium test consists of a horizontal steel rod (diameter: $1 \mathrm{~cm}$, length: $50 \mathrm{~cm}$ ) suspended $40 \mathrm{~cm}$ above a cushioned floor. Mice were placed in the middle of the horizontal rod and released when all 4 paws gripped it, to ensure a stable starting position. This test evaluated the latency to fall (seconds) and the ability of the mice to hold onto the rod for 20 seconds and walk from the middle of the rod to the edge (score 0 : mouse falls down before 20 seconds elapse; score 1: mouse holds onto the rod in the middle part for 20 seconds; score 2: mouse holds onto the rod for 20 seconds and moves from the middle part of the rod). This test was performed 2 times, at 2- to 4-minute intervals between tests.

Coat-hanger test. We used a steel coat hanger (diameter: $2 \mathrm{~mm}$, length: 40 $\mathrm{cm}$ ) divided into 12 segments (length: $5 \mathrm{~cm}$ ) and suspended at a height of $35 \mathrm{~cm}$ from a cushioned surface. The mice were placed in the middle of the hanger, and their behavior was evaluated for a total of 60 seconds. Fall latency, number of movements in 60 seconds, and extreme latency (movement from the middle part of the hanger to the edge) were recorded. The test was concluded when the animal fell down or when 60 seconds elapsed.

Rotarod test. The accelerating rotarod (5-lane accelerating rotarod; LE 8200 , Panlab) was used to measure motor balance and coordination 5 days after spontaneous cannabinoid withdrawal. For 2 consecutive days before the test day, the mice were trained to hold onto the rod at a constant speed (4 rpm) for at least 60 seconds. On the test day, the rod accelerated from 4 to $40 \mathrm{rpm}$ within 5 minutes, and the latency to fall was measured on 10 consecutive trials. Data are expressed as the latency to fall on the first trial (Trial 1) and on the last trial (Trial 10).

\section{Statistics}

Data are presented as the mean \pm SEM. The statistical significance was assessed by ANOVA, followed by a posteriori post-hoc Dunnet's multiple comparison test when appropriate. $P$ values of less than 0.05 were considered significant.

\section{Study approval}

All animal procedures were conducted in accordance with the standard ethical guidelines of the European Communities Directive (86/60-EEC) and approved by the local ethics committee (Comitè Ėtic d'Experimentació Animal, CEEA-PRBB). Animal Welfare Assurance (A5388-01, IACUC approval date June 8, 2009) was granted to our institution by the Office of Laboratory Animal Welfare (OLAW) of the NIH.

\section{Acknowledgments}

We thank Cristina Fernández-Avilés, José María González, and Dulce Real for expert technical assistance, and the staff of the UPF/CRG Flow Cytometry Unit and the Laboratori de Neurofarmacologia for helpful discussion. We thank Wade G. Regehr and Kimberly R. McDaniels of Harvard Medical School for providing the Gabra6cre; $\mathrm{Cb} 1^{\mathrm{f} /+}$ mice. We thank Beat Lutz (Johannes Gutenberg University Mainz, Mainz, Germany) and Giovanni Marsicano (University of Bordeaux, Bordeaux, France) for providing the $\mathrm{Cb} 1 \mathrm{f} / \mathrm{f}$ mice. L. Cutando is a predoctoral fellow of the Fondo de Investigaciones Sanitarias. A. Busquets-Garcia and E. Puighermanal are predoctoral fellows of the Ministerio de Educación. M. Gomis-González is a predoctoral fellow of Fundació La Marató de TV3. This study was supported by grants from the Ministerio de Ciencia e Innovación (BFU2008-03390, to A. Gruart; BFU2008-00899, to J.M. Delgado-García; SAF2007- 
64062 and SAF2011-29864, to R. Maldonado; and SAF2009-07309 and BFU2012-33500, to A. Ozaita); the European Commission (PHECOMP, LSHM-CT-2007-037669, to R. Maldonado); the Instituto de Salud Carlos III (RTA-RETICS, RD06/001/001, to R. Maldonado); the Generalitat de Catalunya (SGR2009-SGR00731, to R. Maldonado); the Fundació La Marató de TV3 (090910, to A. Ozaita); and the ICREA Academia (to R. Maldonado). Partial support from the EU FEDER funds is also acknowledged.

1. Degenhardt L, et al. Toward a global view of alcohol, tobacco, cannabis, and cocaine use: findings from the WHO World Mental Health Surveys. PLoS Med.2008;5(7):e141.

2. Pertwee RG. Emerging strategies for exploiting cannabinoid receptor agonists as medicines. $\mathrm{Br} \mathrm{J}$ Pharmacol. 2009;156(3):397-411.

3. Kano $M$, et al. Endocannabinoid-mediated control of synaptic transmission. Physiol Rev. 2009;89(1):309-380.

4. Munro S, Thomas KL, Abu-Shaar M. Molecular characterization of a peripheral receptor for cannabinoids. Nature. 1993;365(6441):61-65.

5 . Van Sickle MD, et al. Identification and functional characterization of brainstem cannabinoid CB2 receptors. Science. 2005;310(5746):329-332.

6. Maresz K, et al. Modulation of the cannabinoid CB2 receptor in microglial cells in response to inflammatory stimuli. J Neurochem. 2005;95(2):437-445.

7. Bisogno T, Di Marzo V. Cannabinoid receptors and endocannabinoids: role in neuroinflammatory and neurodegenerative disorders. CNS Neurol Disord Drug Targets. 2010;9(5):564-573.

8. Sim-Selley LJ. Regulation of cannabinoid CB1 receptors in the central nervous system by chronic cannabinoids. Crit Rev Neurobiol. 2003;15(2):91-119.

9. Villares J. Chronic use of marijuana decreases cannabinoid receptor binding and mRNA expression in the human brain. Neuroscience. 2007;145(1):323-334.

10. Romero J, et al. Effects of chronic exposure to delta9-tetrahydrocannabinol on cannabinoid receptor binding and $m R N A$ levels in several rat brain regions. Brain Res Mol Brain Res. 1997;46(1-2):100-108.

11. Kawamura Y, et al. The CB1 cannabinoid receptor is the major cannabinoid receptor at excitatory presynaptic sites in the hippocampus and cerebellum. J Neurosci. 2006;26(11):2991-3001.

12. Carey MR, et al. Presynaptic CB1 receptors regulate synaptic plasticity at cerebellar parallel fiber synapses. J Neurophysiol. 2011;105(2):958-963.

13. Thompson RF, Kim JJ. Memory systems in the brain and localization of a memory. Proc Natl Acad SciU S A. 1996;93(24):13438-13444.

14. Tonini R, Ciardo S, Cerovic M. ERK-dependent modulation of cerebellar synaptic plasticity after chronic delta9-tetrahydrocannabinol exposure. J Neurosci. 2006;26(21):5810-5818.

15. Skosnik PD, et al. Cannabis use disrupts eyeblink conditioning: evidence for cannabinoid modulation of cerebellar-dependent learning. Neuropsychopharmacology. 2008;33(6):1432-1440.

16. Edwards CR, et al. Assessment of forebrain-dependent trace eyeblink conditioning in chronic cannabis users. Neurosci Lett. 2008;18(3):264-268.

17. Kishimoto Y, Kano M. Endogenous cannabinoid signaling through the CB1 receptor is essential for cerebellum-dependent discrete motor learning. J Neurosci. 2006;26(34):8829-8837.

18. Steinmetz AB, et al. Examining the effects of former cannabis use on cerebellum-dependent eyeblink conditioning in humans. Psychopharmacology.

Received for publication October 29, 2012, and accepted in revised form March 28, 2013.

Address correspondence to: Andrés Ozaita, Laboratori de Neurofarmacologia, Facultat de Ciències de la Salut i de la Vida, Universitat Pompeu Fabra, Parc de Recerca Biomèdica de Barcelona, C/ Doctor Aiguader 88, 08003 Barcelona, Spain. Phone: 34.93.3160823; Fax: 34.93.3160901; E-mail: andres.ozaita@upf.edu.

2012;221(1):133-141.

19. Edwards CR, Skosnik PD. Cerebellar-dependent learning as a neurobehavioral index of the cannabinoid system. Crit Rev Neurobiol. 2007;19(1):29-57.

20. Stella N. Cannabinoid and cannabinoid-like receptors in microglia, astrocytes, and astrocytomas. Glia. 2010;58(9):1017-1030.

21. Garden GA, Möller T. Microglia biology in health and disease. J Neuroimmune Pharmacol. 2006;1(2):127-137.

22. Nimmerjahn A, Kirchhoff F, Helmchen F. Resting microglial cells are highly dynamic surveillants of brain parenchyma in vivo. Science. 2005; 308(5726):1314-1318.

23. Wake H, Moorhouse AJ, Jinno S. Resting microglia directly monitor the functional state of synapses in vivo and determine the fate of ischemic terminals. J Neurosci. 2009;29(13):3974-3980.

24. Streit WJ. Microglia as neuroprotective, immunocompetent cells of the CNS. Glia.2002;40(2):133-139.

25. Pocock JM, Kettenmann H. Neurotransmitter receptors on microglia. Trends Neurosci. 2007; 30(10):527-535.

26. Färber K, Kettenmann H. Physiology of microglial cells. Brain Res Brain Res Rev. 2005;48(2):133-143.

27. Kaushal V, Schlichter LC. Mechanisms of microgliamediated neurotoxicity in a new model of the stroke penumbra. J Neurosci. 2008;28(9):2221-2230.

28. Yenari MA, Kauppinen TM, Swanson RA. Microglial activation in stroke: therapeutic targets. Neurotherapentics. 2010;7(4):378-391.

29. Kreutzberg GW. Microglia: a sensor for pathological events in the CNS. Trends Neurosci. 1996;19(8):312-318.

30. Colton CA, Wilcock DM. Assessing activation states in microglia. CNS Neurol Disord Drug Targets. 2010;9(2):174-191.

31. Hutcheson DM, Tzavara ET, Smadja C. Behavioural and biochemical evidence for signs of abstinence in mice chronically treated with delta-9-tetrahydrocannabinol. BrJ Pharmacol. 1998;125(7):1567-1577.

32. Thompson RF, Steinmetz JE. The role of the cerebellum in classical conditioning of discrete behavioral responses. Neuroscience. 2009;162(3):732-755.

33. Jiménez-Díaz L, et al. Role of cerebellar interpositus nucleus in the genesis and control of reflex and conditioned eyelid responses. J Neurosci. 2004;24(41):9138-9145

34. Morcuende S, Delgado-Garcia JM, Ugolini G. Neuronal premotor networks involved in eyelid responses: retrograde transneuronal tracing with rabies virus from the orbicularis oculi muscle in the rat. J Neurosci. 2002;22(20):8808-8818.

35. Tikka T, et al. Minocycline, a tetracycline derivative, is neuroprotective against excitotoxicity by inhibiting activation and proliferation of microglia. J Neurosci. 2001;21(8):2580-2588.

36. Bilkei-Gorzo A, et al. Early age-related cognitive impairment in mice lacking cannabinoid CB1 receptors. Proc Natl Acad Sci U S A. 2005; 102(51):15670-15675.
37. Andoh T, Kishi H, Motoki K, et al. Protective effect of IL-18 on kainate- and IL-1 beta-induced cerebellar ataxia in mice. J Immunol. 2008;180(4):2322-2328.

38. Motoki K, et al. The direct excitatory effect of IL-1beta on cerebellar Purkinje cell. Biochem Biophys Res Commun. 2009;379(3):665-668

39. French RA, et al. Expression and localization of $p 80$ and $\mathrm{p} 68$ interleukin-1 receptor proteins in the brain of adult mice. J Neuroimmunol. 1999;93(1-2):194-202.

40. Kim S, Thompson RF. c-Fos, Arc, and stargazin expression in rat eyeblink conditioning. Behav Neurosci. 2011;125(1):117-123.

41. Zhuang S, et al. Effects of long-term exposure to delta9-THC on expression of cannabinoid receptor (CB1) mRNA in different rat brain regions. Brain Res Mol Brain Res. 1998;62(2):141-149.

42. Torres E, et al. Evidence that MDMA ('ecstasy') increases cannabinoid CB2 receptor expression in microglial cells: role in the neuroinflammatory response in rat brain. J Neurochem. 2010;113(1):67-78.

43. Ramírez BG, et al. Prevention of Alzheimer's disease pathology by cannabinoids: neuroprotection mediated by blockade of microglial activation. J Neurosci. 2005;25(8):1904-1913.

44. Albayram O, et al. Role of CB1 cannabinoid receptors on GABAergic neurons in brain aging. Proc Natl Acad Sci U S A. 2011;108(27):11256-11261.

45. Chu LS, et al. Minocycline inhibits 5-lipoxygenase expression and accelerates functional recovery in chronic phase of focal cerebral ischemia in rats. Life Sci. 2010;86(5-6):170-177.

46. Zhao ML, et al. Expression of inducible nitric oxide synthase, interleukin-1 and caspase-1 in HIV-1 encephalitis. J Neuroimmunol. 2001;115(1-2):182-191.

47. Xu-Friedman MA, Harris KM, Regehr WG. Three-dimensional comparison of ultrastructural characteristics at depressing and facilitating synapses onto cerebellar Purkinje cells. J Neurosci. 2001;21(17):6666-6672.

48. Barbour B, Keller BU, Llano I. Prolonged presence of glutamate during excitatory synaptic transmission to cerebellar Purkinje cells. Neuron. 1994;12(6):1331-1343.

49. Marcaggi P, Attwell D. Endocannabinoid signaling depends on the spatial pattern of synapse activation. Nat Neurosci. 2005;8(6):776-781.

50. Chang L, et al. Marijuana use is associated with a reorganized visual-attention network and cerebellar hypoactivation. Brain. 2006;129(pt 5):1096-1112.

51. Ledent C, et al. Unresponsiveness to cannabinoids and reduced addictive effects of opiates in CB1 receptor knockout mice. Science. 1999;283(5400):401-404.

52. Marsicano G, et al. CB1 cannabinoid receptors and on-demand defense against excitotoxicity. Science. 2003;302(5642):84-88.

53. Ozaita A, Puighermanal E, Maldonado R. Regulation of PI3K/Akt/GSK-3 pathway by cannabinoids in the brain. J Neurochem. 2007;102(4):1105-1114.

54. Puighermanal E, et al. Cannabinoid modulation of hippocampal long-term memory is mediated by mTOR signaling. Nat Neurosci. 2009;12(9):1152-1158. 